\title{
Refining pulsar radio emission due to streaming instabilities: Linear theory and PIC simulations in a wide parameter range
}

\author{
Alina C. Manthei ${ }^{1, \star}$, Jan Benáček ${ }^{1}$, Patricio A. Muñoz ${ }^{1}$ and Jörg Büchner ${ }^{1,2}$ \\ ${ }^{1}$ Center for Astronomy and Astrophysics, Technical University of Berlin, 10623 Berlin, Germany \\ e-mail: manthei@physik.uni-bonn.de \\ 2 Max Planck Institute for Solar System Research, 37077 Göttingen, Germany
}

Received: ; accepted:

\begin{abstract}
Context. Several important mechanisms that explain coherent pulsar radio emission rely on streaming (or beam) instabilities of the relativistic pair plasma in a pulsar magnetosphere. However, it is still not clear whether the streaming instability by itself is sufficient to explain the observed coherent radio emission. Due to the relativistic conditions that are present in the pulsar magnetosphere, kinetic instabilities could be quenched. Moreover, uncertainties regarding specific model-dependent parameters impede conclusions concerning this question.

Aims. We aim to constrain the possible parameter range for which a streaming instability could lead to pulsar radio emission, focusing on the transition between strong and weak beam models, beam drift speed, and temperature dependence of the beam and background plasma components.

Methods. We solve a linear relativistic kinetic dispersion relation appropriate for pulsar conditions in a more general way than in previous studies, considering a wider parameter range. In doing so, we provide a theoretical prediction of maximum and integrated growth rates as well as of the fractional bandwidth of the most unstable waves for the investigated parameter ranges. The analytical results are validated by comparison with relativistic kinetic particle-in-cell (PIC) numerical simulations

Results. We obtain growth rates as a function of background and beam densities, temperatures, and streaming velocities while finding a remarkable agreement of the linear dispersion predictions and numerical simulation results in a wide parameter range. Monotonous growth is found when increasing the beam-to-background density ratio. With growing beam velocity, the growth rates firstly increase, reach a maximum and decrease again for higher beam velocities. A monotonous dependence on the plasma temperatures is found, manifesting in an asymptotic behaviour when reaching colder temperatures. A simultaneous change of both temperatures proves not to be a mere linear superposition of both individual temperature dependences. We show that the generated waves are phase-coherent by calculating the fractional bandwidth.

Conclusions. Plasma streaming instabilities of the pulsar pair plasma can efficiently generate coherent radio signals if the streaming velocity is ultra-relativistic with Lorentz factors in the range $13<\gamma<300$, if the background and beam temperatures are small enough (inverse temperatures $\rho_{0} ; \rho_{1} \geq 1$, i.e. $\left.T_{0} ; T_{1} \leq 6 \times 10^{9}\right)$, and if the beam-to-background plasma density ratio $n_{1} /\left(\gamma_{\mathrm{b}} n_{0}\right)$ exceeds $10^{-3}$, which means that $n_{1} / n_{0}$ has to be between 1.3 and $20 \%$ (depending on the streaming velocity)
\end{abstract}

Key words. Pulsars: general - Radio continuum: stars - Plasmas - Instabilities - Relativistic processes

\section{Introduction}

Since the discovery of radio pulsars more than half a century ago in 1967 (see Hewish et al.|1968), a large number of physical models have been proposed to explain their extremely coherent pulsar radio signals (Goldreich \& Julian 1969; Ruderman \& Sutherland 1975; Blaskiewicz et al.||1991; Kramer et al.|2002; Petrova 2009, Beskin 2018; Liu et al. 2019; Philippov et al. 2020). Nevertheless, the scientific community has not yet come to an agreement on a mechanism that can fully explain the observations (Melrose \& Rafat|2017, Beskin|2018).

The current standard model for the (pair) plasma in the pulsar magnetosphere can be summarised as follows (Goldreich \& Julian 1969; Sturrock 1971;, Ruderman \& Sutherland 1975): Due to the fast rotation (with periods from milliseconds to seconds) and the strong magnetic field of the spinning neutron star (typically $10^{11}-10^{13}$ gauss), an electric field has to be generated

\footnotetext{
* Now at: Physikalisches Institut, Rheinische Friedrich-WilhelmsUniversität Bonn, 53115 Bonn, Germany
}

in order to satisfy the ideal frozen-in condition on its surface. This electric field features a very large electrical conductivity and is directed mostly parallel to the magnetic field lines. The screening of this electric field by the plasma already existing in the magnetosphere causes an induced polarisation electric field, forcing the aforementioned plasma to co-rotate with the neutron star. The charge density associated with this induced electric field (and associated co-rotation potential) is called the corotation charge density or the Goldreich-Julian particle density. It typically amounts to $10^{11}-10^{12}$ particles $/ \mathrm{cm}^{3}$ and decreases rapidly with height as far as the so-called light cylinder, where the co-rotation speed of the plasma reaches the speed of light. The light cylinder boundary determines the characteristic size of a pulsar magnetosphere. In the polar cap region of the pulsar magnetosphere, the magnetic field lines are open such that the plasma does not co-rotate there, but rather particles are free to stream and escape along the magnetic field lines. Near the neutron star surface at the polar caps, this escape of particles causes a local plasma depletion on a length scale in the order 
of $10^{4} \mathrm{~cm}$. It leads to a vacuum with an associated strong parallel electric field $\boldsymbol{E} \cdot \boldsymbol{B} \neq 0$ and an associated potential of the order of $10^{12} \mathrm{~V}$. This vacuum region is often called a 'gap', resembling an electrostatic double layer. We note that outside of this gap, $\boldsymbol{E} \cdot \boldsymbol{B}=0$ almost everywhere in the pulsar magnetosphere, such that particles in that region are not accelerated by this mechanism. The charge of the escaping particles depends on the sign of the co-rotation charge; if it is positive near the polar caps, positrons will be accelerated outwards from the neutron star surface while electrons will move in the opposite direction, towards the neutron star. The escaping particles moving along the curved magnetic field lines form the so-called 'primary beam'. They have ultra-relativistic energies and relativistic Lorentz factors $\gamma=\left(1-\beta^{2}\right)^{-1 / 2}$ (where $\beta=v / c$ ) of the order of $\gamma \sim 10^{6}-10^{7}$. This eventually triggers the emission of curvature radiation photons at $\gamma$-ray frequencies. Those photons subsequently undergo pair production, producing a plasma out of electrons and positrons (Sturrock 1971; Cheng \& Ruderman 1977b). Assuming the sign of the co-rotation charge as above, the newly created positrons move away from the neutron star, while the newly created electrons move towards it. These more recently produced particles are called the secondary plasma population, or 'secondary beam'. According to the estimations of Ursov \& Usov (1988), these particles feature relativistic Lorentz factors in the range $\gamma_{\min } \sim 10$ to $\gamma_{\max } \sim 10^{3}-10^{4}$.

Each of these particles is also prone to further emission of $\gamma$-photons due to curvature radiation, followed by a subsequent pair-creation event, at different heights in the gap region. Moreover, synchrotron radiation (due to particle gyromotion in the strong magnetic field) emitted by the secondary beam also contributes to the increasing number of electron-positron pairs since these energetic photons can also undergo pair production. This chain of events leads to the periodic breakdown of the gap vacuum by the generated electrons and positrons when their charge density reaches the Goldreich-Julian density (Goldreich \& Julian 1969). Due to the reduction of the acceleration electric potential by plasma screening in the neighbourhood of the pair production event, particles are no longer accelerated and consequently vacate the region. Since this also implies that no further pair production occurs, the gap region is empty thereafter, and the process of filling it with particles until the renewed attainment of the Goldreich-Julian density may re-start. This periodic 'sparking' process occurs on timescales of microseconds (Ruderman \& Sutherland 1975).

The aforementioned curvature radiation itself, however, cannot be accountable for the observed coherent radio pulses. Coherent radiation means that all particles radiate in phase with each other. An investigation of observational data from six pulsars testing this hypothesis was carried out by Lesch et al. (1998) who discarded it. The observational finding can be reinforced by theoretical arguments. With respect to the emission by the primary beam, the wavelengths of its $\gamma$-ray photons are much smaller than the mean spacing between the plasma particles, such that the curvature radiation by the primary beam is incoherent radiation at much higher frequencies with respect to the radio range. Curvature radiation by the secondary beam, however, was one of the first proposed models to explain pulsar radio emission (Ruderman \& Sutherland 1975). Owing to its lower energy and higher density relative to the primary beam (by a factor of $10^{3}-10^{5}$ ), this beam emits curvature photons with wavelengths much larger than the mean particle spacing, possibly favouring coherent radiation (Beskin et al. 1993). In this case, the plasma frequency and emitted curvature radiation would indeed fall in the radio range. Yet, later theoretical arguments have shown that this hypothesis suffers some deficiencies as well. One of the most important is that it cannot lead to emission with brightness temperatures beyond $>10^{13} \mathrm{~K}$ (Melrose 1978, Appendix B). Hence, this emission is insufficient to explain the observed brightness temperatures of pulsar radio emission, which may reach up to $10^{41} \mathrm{~K}$ (Hankins \& Eilek 2007). The observed emission can only be explained by a coherent mechanism that is characterised by a high brightness temperature (Eilek \& Hankins 2016).

Another possible source for the pulsar radio emission consists in a beam instability (also known as streaming or twostream instability). In this case, the source of free energy is rooted in the relative flow between populations of particles that move at different (mean) velocities.

Kinetic instabilities may generate unstable plasma waves that can be transformed into electromagnetic waves and eventually be observed in the radio regime (Melrose \& Gedalin 1999). At least three scenarios are conceivable for the generation of this (two-)stream instability (Asseo \& Melikidze 1998):

Firstly, an interaction between the primary beam and the secondary plasma is possible (Ruderman \& Sutherland 1975, Cheng \& Ruderman 1977a; Buschauer \& Benford 1977, Arons 1981); however, this process has already been found to be inefficient and cannot account for the generation of the observed radiation. One of the main arguments against it consists in the lack of time for the instability to develop. Usov (1987) pointed out that a time period of $\tau_{\mathrm{I}} \sim 10^{-4}(r / R)^{\frac{3}{2}} \mathrm{~s}$ is necessary for the resulting instability development, assuming a distance $r$ from a pulsar with radius $R$ and provided the parameters of the model in Ruderman \& Sutherland (1975). The plasma particles, by contrast, travel the distance $r$ within $\tau_{0} \sim 3 \cdot 10^{-5}(r / R) \mathrm{s}$, which is smaller than $\tau_{\text {I }}$ for any $r>R$.

Secondly, a different model hypotheses counter-streaming electron and positron populations to be responsible for the observed coherent radio emission (e.g. Ruderman \& Sutherland 1975. Cheng \& Ruderman 1977b; Weatherall 1994). A relative drift between both populations may be caused by the fact that the secondary plasma is exposed to an electric field that arises from the magnetic field curvature and the Goldreich-Julian charge density condition (Cheng \& Ruderman 1977a). However, this kind of instability could easily be quenched by the relativistic temperature of the pulsar magnetosphere plasma (Buschauer \& Benford 1977). Thus, this type of two-stream instability is not likely to be the origin of pulsar radio emission either.

The third possibility, the one that is further investigated in this work, consists of an interaction between two successively emitted plasma bunches, composed of both electrons and positrons. These bunches occur due to the non-stationarity of the plasma emission mechanism in the polar-cap regions, the aforementioned 'spark' events (Ruderman \& Sutherland 1975, Cheng \& Ruderman 1977b). As the filling with new electron-positron pairs takes some time, the previous bunch has already travelled a certain distance outwards when the next one is emitted. The fast particles from the freshly emitted bunch may eventually catch up with the slow ones from the prior particle bunch (Asseo \& Melikidze 1998). The interaction between the particles from the new bunch, forming a particle beam, and those from the former one, considered as the background plasma, can lead to the development of a beam instability, a process proposed in Usov (1987) (see also Ursov \& Usov 1988; Usov 2002). This process underlies the recent studies by Rafat et al. (2019b) whose work we extend and generalise to a broader parameter range.

The pulsar pair plasma, generated by the pair creation process, is relativistic. A relativistically covariant way to take these effects into account is using one-dimensional Maxwell-Jüttner 
(Jüttner 1911) distributions for both the beam and the background plasma (Asseo \& Melikidze 1998). The assumption of one-dimensionality can be adopted in the lower pulsar magnetosphere due to the dominant magnetic field, implying that the drift perpendicular to the magnetic field does not significantly contribute to the particle motion ${ }^{1}$ By means of numerical simulations of the pair creation process, Arendt \& Eilek (2002) found that the typical relativistic bulk flow speed is in the range $p / m c \approx 32-178$ for a magnetic field of $B_{*}=10^{12}$ gauss. The inverse momentum width $p / \Delta p \approx 0.23-0.79$ leads to relativistic temperatures of the particle bunches. They also found that the resulting distribution function is very close to the Maxwell-Jüttner distribution.

It is still unclear, however, what (relativistic) temperatures should be considered. The various pulsar models provide different assumptions here. Weatherall (1994) assume the temperature of the beam to be non-relativistic, while the one of the background is supposed to be highly relativistic in this model. In the case of Rafat et al. (2019b), the same temperature is used for both components. At the distance from the pulsar where the interaction of two bunches takes place, as proposed in the model by Usov (1987), the temperatures of both plasma populations remain unspecified.

Within the same model by Usov (1987), however, it might be possible that the electrons and positrons from a previously emitted bunch of particles could have adiabatically cooled down by the time the fast particles from the succeeding bunch reach them. Choosing the rest frame of the secondly emitted, still hot bunch, that is therefore to be regarded as the background plasma, the firstly emitted and already cooled-down one moves with a relative velocity with respect to the background and can be considered as the beam. A lower beam temperature could lead to a stronger instability compared to the case in which both populations are hot.

It is also conceivable that the temperature has already dropped from the neutron star surface to the interaction region, such that the background and the beam equally face a cool-down, while the ratio of both temperatures persists.

Since the model by Usov $(1987)$ is based on a two-stream instability due to interaction between a previously emitted bunch and the successive one, the densities of the beam and background plasma are assumed to be approximately equal. Rafat et al. (2019b), following Egorenkov et al. (1984), by contrast, conjecture a density ratio of $n_{1} / n_{0}=10^{-3}$ in the background reference frame to be appropriate (corresponding to a weak beam), which makes an investigation of the influence of different densities worthwhile 2

Another important parameter whose influence on the streaming instability has to be understood more thoroughly is the relative velocity of the of beam and background plasma. The exact relative velocity of particles between two bunches, determining the relative drift between beam and background, is not fixed to an explicit value by the presumed model of Usov (1987). We note that the beam-background interaction could take place not only between the fastest particles from the new bunch and the slowest ones from the previously emitted one, but also between

\footnotetext{
1 We note that, by contrast, when the emitted radiation approaches the light cylinder, cyclotron effects might contribute since the electron cyclotron frequency and electron plasma frequency are of the same order of magnitude in this region. Thus, only one-dimensional analysis is no longer adequate in such a case (Luo \& Melrose 2001).

2 We note that in the following, we use the symbols $n_{0}$ and $n_{1}$ to describe the densities of beam and background in their respective reference frames.
}

several groups of particles with intermediate velocities (Asseo $\&$ Melikidze 1998). Thus, the range of possible relative drift speeds between the beam and background plasma can be very broad. Moreover, the more the beam drift speed is reduced, the closer the system gets to a strong-beam model that is defined by $r_{\mathrm{n}}=n_{1} /\left(\gamma_{\mathrm{b}} n_{0}\right) \rightarrow 1$. In our analysis, we orient ourselves by numerical experiments of Arendt \& Eilek (2002) as well as by the parameters used in the analytical relativistic dispersion relations investigated by Rafat et al. (2019b).

Instabilities of relativistic pair plasma beams have been already studied using kinetic plasma simulations (Silva et al. 2003, Tautz et al. 2007; Cottrill et al. 2008; López et al. 2014, D’Angelo et al. 2015; López et al. 2015, Shukla et al.|2015). Only a few studies, however, have assumed relativistic covariant Maxwell-Jüttner distributions, and if so, they were mostly considered to be three-dimensional (Bret et al. 2008, 2010). Only Shalaby et al. (2017) regarded a one-dimensional MaxwellJüttner distribution as more appropriate under the strongly magnetised pulsar conditions. They used it in order to investigate a relativistic two-beam instability with relativistic beam Lorentz factors of $\gamma_{\mathrm{b}} \leq 4$. Beyond that, following up on these results, Shalaby et al. (2018) also analysed higher beam velocities $\left(\gamma_{\mathrm{b}}=100\right)$ for an inhomogeneous background.

A discussion of the influence of the adopting this MaxwellJüttner distribution function and its associated relativistic effects on the wave dispersion, with a view to pulsar radio emission, has already been carried out by Rafat et al. (2019b). Their investigations led to the conclusion that the above mentioned beam-driven instabilities do not suffice as a primary source for the observed pulsar radiation. However, this might be due to the limited range range of plasma parameters for which Rafat et al. (2019b) carried out their calculations.

Beyond that, Rahaman et al.(2020) recently solved a hot relativistic dispersion relation appropriate for a pulsar plasma magnetosphere as well. Similarly to our study, they also considered the streaming instability due to the overlap of successively emitted plasma bunches within the Usov (1987) model, but additionally taking the pulsar magnetosphere geometry into account and comparing among several pulsar emission models. They found the most efficient mechanism that should be responsible for the highest growth rates among those models and the expected emission height. Different from our work, however, they did not carry out numerical simulations to confirm the solutions of their dispersion relations.

Here, we re-investigate the question whether streaming instabilities may cause the observed pulsar radio emission by extending the investigations by Rafat et al. (2019b) and their range of considered plasma parameters. As far as we know, for the first time, we investigate the dependence of wave dispersion branches and instability growth rates on a wide range of plasma properties, in order to obtain stronger constraints on the possible conditions for an efficient radio-pulsar emission due to streaming instabilities. Those investigated parameters include the temperature of the background and beam, beam velocity, and the beambackground density ratio. Beyond the scope of previous studies, we additionally compare our analytical linear dispersion relation solutions with suitable kinetic particle-in-cell (PIC) simulations.

The outline of this paper is the following. First, we give a short summary of the theoretical basis (Sect. 2). Then, we state the methods used for solving the dispersion relations, and we describe our simulation setup (Sect. 3). Next, we conduct several parametric studies in order to constrain the parameter regime where instabilities could be able to grow and generate plasma 
waves (Sect.47. In Sect.5, we compare the analytical dispersion relation solutions and the kinetic simulation results.

\section{Dispersion relation for a Maxwell-Jüttner velocity distribution in a pulsar pair plasma}

In order to cover a wide range of plasma parameters, this section is devoted to the derivation of an appropriate generalised kinetic dispersion relation based on the model by Ursov \& Usov (1988). Here we consider a pair plasma composed of electrons with density $n_{-}$and positrons with density $n_{+}$. Within the bunch model by Ursov \& Usov (1988), we assume a 'background' (denoted with subscript 0 ) at rest and a 'beam' (denoted with subscript 1) drifting outwards parallel to the magnetic field at a relative speed of $v_{\mathrm{b}}=\beta_{\mathrm{b}} c$. We note that we do not intend to investigate instabilities caused by the relative streaming of both populations due to the magnetic field curvature (Cheng \& Ruderman 1977a). Hence we neglect the small difference between $n_{-}$and $n_{+}$that the latter would cause. Instead, within the model by Ursov \& Usov (1988), each background and beam plasma population contains the same number of electrons and positrons. Therefore, they would simply be equal to $n_{0}$ in the reference frame of the background plasma. Similarly, the electrons and positrons in the beam feature a density $n_{1}$ in their own reference frame. All our calculations are performed in the reference frame of the background such that the beam density is equal to $n_{1} / \gamma_{\mathrm{b}}$ in this reference frame, with the relativistic Lorentz factor $\gamma_{\mathrm{b}}=\left(1-\beta_{\mathrm{b}}^{2}\right)^{-1 / 2}$. Such a plasma system can be described by different distribution functions. At the beginning, the discussion below does not assume a specific form of the distribution function. The specific relativistic plasma dispersion function that is used in our study is described after that.

Generally, the linear plasma response to small perturbations (linear dispersion theory) can be described by the dielectric tensor $K_{i j}(\omega, \boldsymbol{k})$. By equating its determinant to zero, a dispersion relation $\omega=\omega(\boldsymbol{k})$ can be obtained that determines all the properties of linear plasma electrostatic wave modes. In general, both frequency $\omega=\omega_{\mathrm{r}}+i \omega_{\mathrm{i}}$ and wave vector $\boldsymbol{k}=\boldsymbol{k}_{\mathrm{r}}+i \boldsymbol{k}_{\mathrm{i}}$ can be complex numbers. The question whether the wave growth is chosen to be considered as purely temporal or purely spatial is determined by the specific circumstances and also by practical aspects of the particular application. Depending on the physical problem under consideration, the dielectric tensor might be simplified by appropriate choices (Sturrock 1958, Weatherall 1994), facilitating the calculations. For our purpose in this work, the frequency $\omega$ is chosen to be imaginary while keeping the wavenumber $k$ real. This implies that $\omega_{\mathrm{i}}$ characterises the wave growth. For $\omega_{\mathrm{i}}>0$, the wave grows exponentially, being the hallmark of a plasma instability, whereas the wave is damped for $\omega_{\mathrm{i}}<0$.

Following Gedalin et al. (1998); Melrose \& Gedalin (1999), we calculate the dielectric tensor $K_{i j}$ in a three-dimensional Cartesian geometry, assuming a background magnetic field oriented in the direction denoted as 3 . We further assume that both wave propagation and field fluctuations only occur in direction parallel to the magnetic field, corresponding to a onedimensional plasma model. This has proved an appropriate approximation to describe streaming instabilities in a pair plasma in the pulsar magnetosphere emission region, mainly justified by the strong magnetisation in this region (Weatherall 1994). Furthermore, the wave frequency is assumed to be much smaller than the corresponding cyclotron frequency, meaning $\omega \ll$ $\Omega_{\mathrm{e}^{ \pm}} /\langle\gamma\rangle$ with $\Omega_{\mathrm{e}^{ \pm}}=e B / m_{\mathrm{e}}$. Hence cyclotron resonance effects can be neglected.

Under the aforementioned assumptions, the dispersion relation simplifies considerably. Only the $K_{33}$ component parallel to the magnetic field (out of six independent components) does not vanish. Thereby, the full dispersion relation $\left|K_{i j}(\omega, k)\right|=0$ becomes

$K_{33}=1-\frac{\omega_{\mathrm{p}}^{2}}{\omega^{2}} \cdot z^{2} W(z)=0$,

where $\omega_{\mathrm{p}}$ is the plasma frequency of both populations, including a relativistic correction due to the beam Lorentz factor:

$\omega_{\mathrm{p}}^{2}=\frac{e^{2}}{\epsilon_{0} m_{\mathrm{e}}}\left(2 n_{0}+2 \frac{n_{1}}{\gamma_{b}}\right)$.

In the above equation, $e$ denotes the elementary charge, $\epsilon_{0}$ stands for the vacuum electric permittivity, and $m_{\mathrm{e}}$ is the electron (or positron) mass. We note that $\omega_{\mathrm{p}}$ is the physical fundamental oscillation frequency in a pair plasma, different from $\omega_{\mathrm{pe}}=e^{2} n_{-} /\left(m_{\mathrm{e}} \epsilon_{0}\right)=\omega_{\mathrm{p}} / \sqrt{2}$ with $n_{-}=n_{0}+n_{1}$ (Stenson et al. 2017). The parameter $z=\omega /(k c)$ is used to indicate the phase velocity of a wave with frequency $\omega$ and wavenumber $k$. The socalled relativistic plasma dispersion function $W(z)$ in Eq. (1) is defined as

$W(z)=W_{0}(z)+r_{\mathrm{n}} \gamma_{\mathrm{b}} W_{1}(z)$

where $r_{\mathrm{n}}=n_{1} /\left(\gamma_{\mathrm{b}} n_{0}\right)$ describes the ratio of densities between beam and background plasma in the rest frame of the background. The respective background $\left(W_{0}\right)$ and beam $\left(W_{1}\right)$ relativistic dispersion functions can be written in the following way:

$$
\begin{array}{r}
W_{\alpha}(z)=\frac{1}{n_{\alpha}} \int_{-\infty}^{\infty} \mathrm{d} u \frac{\mathrm{d} g_{\alpha}(u) / \mathrm{d} u}{\beta-z}=\frac{1}{n_{\alpha}} \int_{-1}^{1} \mathrm{~d} \beta \frac{\mathrm{d} g_{\alpha}(u) / \mathrm{d} \beta}{\beta-z} \\
\alpha=0,1
\end{array}
$$

where $u=\beta /\left(1-\beta^{2}\right)^{\frac{1}{2}}$. This transcendental function has a singularity at $\beta=z$ that has to be taken care of by applying Cauchy's integral formula. Further properties of this function are discussed in Godfrey et al. (1975); Rafat et al. (2019b).

The relativistic function (Eq. (3) ) and so the dispersion relation (Eq. (1) require the specification of an appropriate distribution function $g_{\alpha}$. In order to account for possible relativistic temperatures as well as relativistic bulk flow velocities of both beam and background plasma, we model their velocity distribution functions $g_{\alpha}$ as relativistically covariant Maxwell-Jüttner distributions. These velocity distributions depend on both the inverse temperature in units of the particle rest energy, denoted as $\rho=m_{\mathrm{e}} c^{2} /\left(k_{\mathrm{B}} T\right)$, as well as on the relativistic Lorentz factor $\gamma=\left(1-\beta^{2}\right)^{-1 / 2}$. This way, plasmas with relativistic temperatures are characterised by $\rho<1$ while relativistic plasma flows have the property $\gamma \gg 1$.

The resulting Maxwell-Jüttner velocity distribution function of the entire plasma is composed of the individual distributions for each plasma population. In the reference frame of the background, it can be written as (Rafat et al.2019b):

$$
\begin{aligned}
g(u) & =g_{0}(u)+g_{1}(u) \\
g_{0}(u) & =n_{0} \cdot \frac{\exp \left(-\rho_{0} \gamma\right)}{2 K_{1}\left(\rho_{0}\right)} \\
g_{1}(u) & =\frac{n_{1}}{\gamma_{\mathrm{b}}} \cdot \frac{\exp \left(-\rho_{1} \gamma_{\mathrm{b}} \gamma\left(1-\beta \beta_{\mathrm{b}}\right)\right)}{2 K_{1}\left(\rho_{1}\right)}
\end{aligned}
$$

where $K_{1}$ denotes the Macdonald function of first order (also known as modified Bessel function of the second kind). 
The source of free energy for kinetic streaming instabilities can be traced back to a positive slope $\mathrm{d} g(u) / \mathrm{d} u>0$ for $u>0$ of the joint velocity distribution of Eq. (4) (Baumjohann \& Treumann 1997). Since there are more particles with higher velocities than the wave phase speed in the positive slope region, a wave with $\beta<\beta_{\mathrm{b}}$ is able to gain more energy than it loses by interactions with lower-momentum particles. Such a positive slope is possible if the centre (bulk flow speed) of the beam distribution (6) in velocity space is sufficiently separated from the background distribution function (5). This implies the existence of a well-defined minimum in the joint velocity distribution (4). This condition can be formally stated by means of the so-called Penrose criterion, also known as 'separation condition' (between the beam and background distributions). In the model presented here, it predicts the following minimum beam drift speed that would be necessary for the system to become unstable (Melrose 1986; Rafat et al. 2019b):

$$
\begin{gathered}
\gamma_{\mathrm{b}, \min } \approx 7.8\left(\frac{1}{r_{\mathrm{n}} r_{\rho} r_{\mathrm{K}}}\right)^{0.076}\left(\frac{1}{\rho_{0}}\right)^{1.07}, \\
r_{\rho}=\frac{\rho_{1}}{\rho_{0}}, \quad r_{\mathrm{K}}=\frac{K_{1}\left(\rho_{0}\right)}{K_{1}\left(\rho_{1}\right)} .
\end{gathered}
$$

Besides the growth rates, corresponding to the imaginary parts of the frequency, the fractional bandwidth of the growing modes can be examined by studying the real part of the frequency. The bandwidth is defined as the frequency range between the two values of $\omega_{\mathrm{r}}$ for which the growth rate $\left(\omega_{\mathrm{i}}\right)$ has decreased to half of its maximum value (FWHM). To obtain a dimensionless quantity, a division by $\omega_{\mathrm{r}}$, where the maximum of $\omega_{\mathrm{i}}$ occurs, is performed. This yields the fractional bandwidth $\Delta \omega_{\mathrm{r}} / \omega_{\mathrm{r}}\left(\omega_{\mathrm{i}, \max }\right)$. This quantity determines the maximum value of the growth rate for which the random phase mixing approximation is valid. If the growth rate value exceeds the value of the fractional bandwidth, the unstable wave mode is phase-coherent.

One way to quantify the efficiency of the wave growth is via the growth factor $G$, a quantity that, in the case of purely temporal growth, is defined as (Melrose 1986):

$G=\omega_{\mathrm{i}} \Delta t$,

where $\Delta t$ is the growth time. Within the Ruderman \& Sutherland (1975) sparking model, it can be interpreted as the time span between two pair creation events. The boundary between efficient and inefficient growth occurs at $G=1$. Thus, a necessary condition for the instability to be effective is $G>1$.

\section{Methods}

The linear kinetic dispersion relation of Eq. (1) can be solved numerically, and its solutions can be compared to results of particle-in-cell (PIC) code simulations. Both methods are described in this section.

\subsection{Numerical solution the dispersion relation}

Our aim is to solve the linear kinetic dispersion relation (1) in a wider range of plasma parameters and by assuming fewer simplifications than previous studies (cf. Rafat et al. (2019b)). Rafat et al. (2019b) found the roots of the dispersion relation (1) by using an expansion of $K_{33}$ about $\omega_{\mathrm{r}}$ and $k_{\mathrm{r}}$ to the first order in $\omega_{\mathrm{i}}$ and $k_{\mathrm{i}}$. This approach assumes $\omega_{\mathrm{i}} / \omega_{\mathrm{r}} \ll 1$ and $k_{\mathrm{i}} / k_{\mathrm{r}} \ll 1$.
In contrast, we do not make such an assumption, allowing for arbitrary values of $\omega_{\mathrm{i}}$. To do so, first we sample independently both real and imaginary part of the complex function $K_{33}$ on a grid in the plane $\left(\omega_{\mathrm{r}}, \omega_{\mathrm{i}}\right)$. This sampling is carried out for a given $k_{0}$ where we expect to find a root of the dispersion relation. Then, we trace the contour levels of the real and imaginary parts of $K_{33}$ that are equal to 0 for the chosen $k_{0}$. The solutions of Eq. (1) will thus be given by the intersections of those contours where both real and imaginary parts vanish simultaneously. A first-order approximation for those intersections is found by a geometrical method based on determining the intersections of the piecewise straight lines joining each grid point of the ' 0 '-contour levels discretised onto the grid $\left(\omega_{\mathrm{r}}, \omega_{\mathrm{i}}\right)$. This method leads to intersections that are accurate above the grid resolution level $\Delta \omega_{\mathrm{r}}=\left(\omega_{\mathrm{r}, \max }-\omega_{\mathrm{r}, \min }\right) /\left(n_{\omega}-1\right), \Delta \omega_{\mathrm{i}}=\left(\omega_{\mathrm{i}, \max }-\omega_{\mathrm{i}, \min }\right) /\left(n_{\omega}-1\right)$, where $n_{\omega}=50$, for an initially given $\omega_{\mathrm{r} / \mathrm{i} \text {,min }}$ and $\omega_{\mathrm{r} / \mathrm{i} \text {,max }}$. Subsequently, a high-accuracy intersection is found by using the previous initial intersection as an initial guess for the multidimensional root finding function named root of the module optimise in the Python library SciPy, which uses the nonlinear least squares Levenberg Marquardt algorithm (Levenberg 1944, Marquardt 1963). This algorithm combines the GaussNewton algorithm and the method of gradient descent. Its tolerance for convergence amounts to $10^{-12}$. If this root finding algorithm fails to converge, a different approach is used: the modified Powell's hybrid method (Powell 1964) with a tolerance for convergence of $10^{-6}$. The intersection that is eventually discovered, $\left(\omega_{\mathrm{r} 0}, \omega_{\mathrm{i} 0}, k_{0}\right)$, represents a root of Eq. (11). Once this initial intersection has been found, a solution for the next point $k_{1}=k_{0}+\Delta k$ with $\Delta k=\left(k_{\max }-k_{\min }\right) /\left(n_{k}-1\right), n_{k}=2000$ (given the initially set range of $\left.k_{\max }-k_{\min }\right)$ is looked for by using as initial guess a linear extrapolation (adapted to the resolution in $k)$ of $\left(\omega_{\mathrm{r} 0}, \omega_{\mathrm{i} 0}\right)$ and the same root finding algorithm. This leads to the subsequent triple of $\left(\omega_{\mathrm{r} 1}, \omega_{\mathrm{i} 1}, k_{1}\right)$. This process continues iteratively until two sufficiently smooth curves, $\left(\omega_{\mathrm{r}}, k\right)$ and $\left(\omega_{\mathrm{i}}, k\right)$, are found for a given range of $k$, which constitute the frequency and growth rate of a specific wave mode. Numerically, these curves represent one solution of the dispersion relation for each initial intersection $\left(\omega_{\mathrm{r} 0}, \omega_{\mathrm{i} 0}, k_{0}\right)$. This algorithm was validated by reproducing the relativistic dispersion relation curves of Godfrey et al. (1975), in particular for arbitrary magnitude of $\omega_{\mathrm{i}}$ with respect to $\omega_{\mathrm{r}}$, and some results of Rafat et al. (2019a).

Due to the high precision of the used root finding methods, the uncertainties on the results are very small. This implies that those uncertainties are not visible in the following plots. For the plots showing the maximum growth rates, the uncertainties are enhanced by the limited number of steps in $k$ for which a solution is found. This implies that the actual maximum might be missed due to a too large step size. However, the combination of both uncertainties is not visible in the corresponding plots either.

A comparison between numerical solutions and results from simulations is possible by considering the maximum values obtained for the respective growth rates. In addition, to have a comparable quantity that considers the full range of wave growth in $k$-space, it is possible to define an 'integrated growth rate' $\Gamma$ : It is defined in such a way that it reveals information about the total electric energy growth:

$\Gamma=\frac{\int_{z} E(z) \omega_{\mathrm{i}}(z) \mathrm{d} z}{\int_{z} E(z) \mathrm{d} z}$.

The above integral is evaluated for all phase velocities $z$ in the range of positive wave growth. For comparison with simulation results, each growth rate is multiplied by the relativistic 
kinetic energy corresponding to the respective phase velocity, $E(z)=(\gamma-1) m_{\mathrm{e}} c^{2}, \gamma=\sqrt{1+z^{2}}$, and the integral over all kinetic energies is used as a normalisation. Both of the above mentioned quantities, the maximum and integrated growth rates, are considered when comparing the predictions of the linear dispersion theory with the numerical simulation results (Sect.4).

\subsection{PIC simulations}

We simulated the evolution of streaming instabilities for a wide range of different parameters (beam Lorentz factor, beam and background temperatures, and beam-to-background density ratio) that might be characteristic for the plasma of pulsar magnetospheres. For the fully kinetic simulations of streaming instabilities we utilise the fully kinetic PIC code ACRONYM (Kilian et al. 2012 ${ }^{3}$ ACRONYM contains some features that allow for the elimination of numerical problems and/or instabilities that might occur in kinetic simulations of relativistic plasmas such as those of pulsar magnetospheres. In particular, ACRONYM can properly handle highly relativistic velocities of particles and wave propagation in relativistic plasmas. For our purpose, we use the one-dimensional version of the code. The code employs the Cole-Kärkkäinen (CK) non-standard finite difference discretisation scheme (NSFD) for the Maxwell field solver (Cole 1997; Kärkkäinen et al. 2006). This Maxwell solver minimises the numerical dispersion of light waves and, thus, any spurious numerical Cherenkov radiation that might occur in relativistically drifting plasma populations, as it is the case for pulsar plasmas. Beyond that, our PIC code also utilises a recently developed and specialised shape function to reduce the numerical Cherenkov radiation, that is to say a higher-order interpolation scheme known as the 'Weighting with Time-step dependency' (WT) scheme (Lu et al. 2020) in its fourth order version. To avoid any influence of the choice of shape function in a PIC code on the conservation properties of the system, we choose a charge-conserving current density deposition scheme following Esirkepov (2001). This approach allows us to use the WT scheme without affecting energy conservation. We verified that the total energy conservation of this setup is good, while providing an exact conservation of momentum and charge and suppressing the numerical Cherenkov radiation.

The electron-positron pair plasma is initialised by onedimensional Maxwell-Jüttner velocity distribution functions given by Eq. 5 for the background and by Eq. 6 for the beam. Particles are generated using the rejection method in an interval that is larger than the expected velocity range of the distribution function. We compared the resulting initial particle distribution functions with the analytical ones and found a good agreement. We also tested the convergence using higher numbers of particles, finding that the number of particles per cell as given in Table 1 is sufficient to accurately describe the initial distribution function.

We conducted 15 simulations to cover the range of the investigated parameters. Table 1 summarises the main numerical and physical parameters of the simulations. In our simulations, the time step is $\omega_{\mathrm{p}} \Delta t=0.03519$ and the (normalised) grid size to the particle skin depth corresponds to $\Delta x \omega_{\mathrm{p}} / c=0.07108$ (or $\left.d_{\mathrm{e}} / \Delta x=14.07\right)$.

The Debye length is estimated as

$\lambda_{\mathrm{D}}=\left(\frac{1}{\lambda_{0}}+\frac{1}{\lambda_{1}}\right)^{-\frac{1}{2}}$,

\footnotetext{
3 http://plasma.nerd2nerd.org/papers.html
}

where $\lambda_{0}, \lambda_{1}$ are the background and beam Debye lengths. This definition can be expressed in terms of the relativistic background and beam 'sound' speeds, $c_{\mathrm{s} 0}$ and $c_{\mathrm{s} 1}$, as (Diver \& Laing 2015):

$\lambda_{\mathrm{D}}=\left[\frac{\omega_{\mathrm{p}}^{2}}{n_{0}+r_{\mathrm{n}}}\left(n_{0} \frac{1}{c_{\mathrm{s} 0}^{2}}+r_{\mathrm{n}} \frac{1}{c_{\mathrm{s} 1}^{2}}\right)\right]^{-\frac{1}{2}}$,

which, for the Maxwell-Jüttner distribution, becomes:

$$
c_{\mathrm{s} 0,1}^{2}=\frac{c^{2}}{G_{1 \mathrm{D}}} \frac{\mathrm{d} G_{1 \mathrm{D}}}{\mathrm{d} \rho_{0,1}}\left(\rho_{0,1} \frac{\mathrm{d} G_{1 \mathrm{D}}}{\mathrm{d} \rho_{0,1}}+\frac{1}{\rho_{0,1}}\right)^{-1},
$$

with

$$
G_{1 \mathrm{D}}\left(\rho_{0,1}\right)=\frac{K_{2}\left(\rho_{0,1}\right)}{K_{1}\left(\rho_{0,1}\right)},
$$

where the function $G_{1 \mathrm{D}}$ applies to a one-dimensional velocity distribution with two degrees of freedom (corresponding to electrons and positrons, respectively) and the adiabatic index $\kappa=4 / 2$. For the three-dimensional case, frequently used in astrophysics, $G_{3 \mathrm{D}}=K_{3}(\rho) / K_{2}(\rho)$ has six degrees of freedom and an adiabatic index of $\kappa=8 / 6$. This sound speed approaches $c_{\mathrm{s}} \rightarrow c$ for $\rho \rightarrow 0$ (ultra-relativistically hot plasma). For example, the sound speed is $c_{\mathrm{s}}=0.827 c$ for $\rho=1$, while $c_{\mathrm{s}}=0.171 c$ for $\rho=100$. We note that $c_{\mathrm{s}} \rightarrow \sqrt{k_{\mathrm{B}} T / m_{\mathrm{e}}}$, where $k_{\mathrm{B}}$ is the Boltzmann constant, for $\rho \rightarrow \infty$, which means that the 'sound' speed becomes the standard thermal speed under non-relativistic conditions. In our simulations, this normalised Debye length varies in the range $\lambda_{\mathrm{D}} / \Delta x=2.74-12.8$ for our limiting cases (maximal and minimal temperatures), providing at least (in case of $\left.\rho_{0}=\rho_{1}=100\right) 2740$ background particles and 712 beam particles per Debye length.

The one-dimensional simulation box extends along the first (or $x$-) direction, that is to say the direction of the pulsar magnetic field. Periodic boundary conditions are applied.

Because of the strong magnetic field that dominates the pulsar magnetosphere, a one-dimensional domain with a onedimensional velocity distribution is sufficient to describe its plasma dynamics. This also implies that magnetic effects are suppressed in our simulation, and all quantities have only onedimensional profiles. As the particle current is always along the $x$-direction due to its initial velocity distribution, it creates electric currents and an electric field solely in this direction. An electric field only in the $x$-direction does not induce any nonzero time derivative of the magnetic field, meaning that $\partial \mathbf{B} / \partial t=$ $-\nabla \times \mathbf{E}=-\nabla \times\left(E_{x}, 0,0\right)=\left(0, \partial E_{x} / \partial z,-\partial E_{x} / \partial y\right)=(0,0,0)$, since the spatial derivatives along the $y$ - and $z$-directions of an arbitrary function are always zero in a one-dimensional simulation. This implies that the system behaves in the same way as for $\delta B / B \rightarrow 0$ and particles are not influenced by any arbitrary magnetic field as its direction coincides with the direction of particle movement (for further details see Melrose (2017), Sections 4.3 and 4.4)).

The background contains $N_{0}$ electrons and $N_{0}$ positrons per cell, while the beam is composed of $N_{1}$ electrons and $N_{1}$ positrons per cell. The background and beam populations are individually charge-neutral in each cell, independent of the respective other population. We tested the total energy conservation of the code, the generation of wave harmonics, the resolution of the frequency and of the wave vector for the analysed relativistic plasma regime. The results of those tests led us to choose either $10^{3}$ or $10^{4}$ background particles per cell per species. Similarly, we found that for good numerical stability, the number of beam 
Table 1. Synopsis of the relevant numerical and physical parameters underlying the PIC simulations: Simulation reference number, beam Lorentz factors $\gamma_{b}$, inverse background $\left(\rho_{0}\right)$ and beam $\left(\rho_{1}\right)$ temperatures, simulation length $L$ in number of grid cells $(\Delta)$, number of particles per cell per species (electrons and positrons) of the background $N_{0}$ and of the beam $N_{1}$, beam-to-background particle density ratio $r_{\mathrm{n}}$, integrated growth rates from PIC simulations $\Gamma$, maximum found growth rate in $k$-space $\omega_{\mathrm{i}, \mathrm{max} \text {,sim }}$, and the fractional bandwidth $\Delta \omega_{\mathrm{r}} / \omega_{\mathrm{r}}$.

\begin{tabular}{crrrrrrlcrr}
\hline \hline $\begin{array}{c}\text { Sim. } \\
\text { No. }\end{array}$ & $\gamma_{\mathrm{b}}$ & $\rho_{0}$ & $\rho_{1}$ & $\mathrm{~L}[\Delta]$ & $N_{0}$ & $N_{1}$ & \multicolumn{1}{c}{$r_{\mathrm{n}}$} & $\Gamma / \omega_{\mathrm{p}}$ & $\omega_{\mathrm{i}, \mathrm{max}, \mathrm{sim} / \omega_{\mathrm{p}}}$ & $\Delta \omega_{\mathrm{r}} / \omega_{\mathrm{r}}$ \\
\hline 1 & 26 & 1 & 1 & $10^{4}$ & $10^{4}$ & 260 & 0.001 & $(5.1 \pm 0.5) \times 10^{-4}$ & $(8.7 \pm 0.2) \times 10^{-4}$ & $0.12 \pm 0.02$ \\
2 & 60 & 1 & 1 & $10^{4}$ & $10^{4}$ & 600 & 0.001 & $(9.6 \pm 0.6) \times 10^{-4}$ & $(1.28 \pm 0.09) \times 10^{-3}$ & $0.09 \pm 0.02$ \\
3 & 103 & 1 & 1 & $10^{4}$ & $10^{4}$ & 1030 & 0.001 & $(1.07 \pm 0.04) \times 10^{-3}$ & $(1.34 \pm 0.02) \times 10^{-3}$ & $0.09 \pm 0.01$ \\
4 & 26 & 1 & 1 & $10^{5}$ & $10^{3}$ & 1000 & 0.0385 & $(3.68 \pm 0.03) \times 10^{-3}$ & $(5.24 \pm 0.22) \times 10^{-3}$ & $0.38 \pm 0.06$ \\
5 & 60 & 1 & 1 & $10^{5}$ & $10^{3}$ & 1000 & 0.0167 & $(3.72 \pm 0.09) \times 10^{-3}$ & $(4.61 \pm 0.06) \times 10^{-3}$ & $0.29 \pm 0.02$ \\
6 & 103 & 1 & 1 & $10^{5}$ & $10^{3}$ & 1000 & 0.00971 & $(2.80 \pm 0.02) \times 10^{-3}$ & $(3.40 \pm 0.08) \times 10^{-3}$ & $0.23 \pm 0.04$ \\
7 & 26 & 1 & 10 & $10^{4}$ & $10^{4}$ & 260 & 0.001 & $(2.76 \pm 0.04) \times 10^{-3}$ & $(3.18 \pm 0.06) \times 10^{-3}$ & $0.17 \pm 0.03$ \\
8 & 26 & 1 & 100 & $10^{4}$ & $10^{4}$ & 260 & 0.001 & $(3.31 \pm 0.06) \times 10^{-3}$ & $(3.54 \pm 0.09) \times 10^{-3}$ & $0.19 \pm 0.06$ \\
9 & 26 & 10 & 10 & $10^{4}$ & $10^{4}$ & 260 & 0.001 & $(7.16 \pm 0.07) \times 10^{-3}$ & $(6.83 \pm 0.05) \times 10^{-3}$ & $0.08 \pm 0.02$ \\
10 & 26 & 100 & 100 & $10^{4}$ & $10^{4}$ & 260 & 0.001 & $(7.02 \pm 0.07) \times 10^{-3}$ & $(6.15 \pm 0.04) \times 10^{-3}$ & $0.09 \pm 0.04$ \\
11 & 26 & 10 & 1 & $10^{4}$ & $10^{4}$ & 260 & 0.001 & $(4.28 \pm 0.06) \times 10^{-3}$ & $(4.43 \pm 0.06) \times 10^{-3}$ & $0.07 \pm 0.01$ \\
12 & 26 & 100 & 1 & $10^{4}$ & $10^{4}$ & 260 & 0.001 & $(5.10 \pm 0.06) \times 10^{-3}$ & $(5.39 \pm 0.06) \times 10^{-3}$ & $0.11 \pm 0.05$ \\
13 & 26 & 1 & 1 & $10^{4}$ & $10^{3}$ & 2600 & 0.1 & $(6.06 \pm 0.05) \times 10^{-3}$ & $(7.89 \pm 0.11) \times 10^{-3}$ & $0.65 \pm 0.14$ \\
14 & 26 & 1 & 1 & $10^{4}$ & $10^{3}$ & 7800 & 0.3 & $(10.33 \pm 0.09) \times 10^{-3}$ & $(12.37 \pm 0.05) \times 10^{-3}$ & $0.81 \pm 0.20$ \\
15 & 26 & 1 & 1 & $10^{4}$ & $10^{3}$ & 26000 & 1 & $(15.79 \pm 0.04) \times 10^{-3}$ & $(17.06 \pm 0.10) \times 10^{-3}$ & $1.16 \pm 0.18$ \\
\hline
\end{tabular}

particles per cell has to be $>100$ for the lower values $(\sim 26)$ of the beam Lorentz factor $\gamma_{\mathrm{b}}$. For $\gamma_{b} \sim 100$, however, the beam distribution function becomes very broad in the Lorentz factor $\gamma$, such that some particles in the tail of the beam distribution function reach $\gamma>10^{3}$. Hence, we used at least $10^{3}$ beam particles per cell in this case. We note that the number of particles in the beam depends on its Lorentz factor $\gamma_{\mathrm{b}}$ and is thus given as $N_{1}=r_{\mathrm{n}} \gamma_{\mathrm{b}} N_{0}$, such that, for a density ratio of $r_{\mathrm{n}}=1$ and for example $N_{0}=10^{3}$ as in simulation 15 , the number of beam particles $N_{1}=26 \cdot N_{0}=26 \times 10^{3}$ does not match, but rather exceeds the number of background particles in the background reference frame. On the other hand, the beam density, and thus the number of beam particles (assuming an equal ratio of physical to numerical particles), remains equal in the beam reference frame.

The duration of the simulations depends on the time of the instability onset and its growth rates that are different for each case. The total simulation time $\omega_{\mathrm{pe}} \Delta T$ thus varies in the range between 2000 and 15000. A typical simulation run consumes 5000 CPU hours. The simulation domain size spans either $10^{4}$ or $10^{5}$ grid cells (see $L[\Delta]$ in Table 1 All our simulations have a spectral resolution that is high enough to describe the wave vector space accurate enough.

The integrated growth rates $\Gamma$ obtained from our simulation, which shall be compared to the numerical integrated growth rates, are estimated from the energy of the electric field in the time period from the onset of the exponential growth until the start of saturation. During this time period, the energy evolution can be fitted by the function $F(t)=E_{0}+E_{1} \mathrm{e}^{2 \Gamma t}$, where $E_{0}, E_{1}, \Gamma$ are arbitrary fit constants. From this fit, the growth rates $\Gamma$ of the total electric energy were calculated. For a better comparison with linear theory, we also analysed the growth rates as a function of the wavenumber $k$ in order to compare with the analytically obtained maximum growth rates $\omega_{\mathrm{i}}$. We transformed the profile of the electric field in the configuration space $\boldsymbol{E}(x)$ into the wavenumber space $\boldsymbol{E}(k)$ by applying a Fast Fourier Transform (FFT) at each time step. Then, we computed the time evolution of the electric field $\boldsymbol{E}(k, t)$ for 50 equidistant wavenumbers in the range $k c / \omega_{\mathrm{p}} \in(0,2.5)$. Subsequently, we performed an exponential fit of the function $f(t)=e_{0}+e_{1} \mathrm{e}^{\Gamma t}$ for each of them in the same time window ${ }^{4}$ The maximum growth rate $\omega_{i \text {,max,sim }}$ is then selected as the largest growth rate that has by found by this procedure.

Two different kinds of uncertainty may occur when estimating $\Gamma$ and $\omega_{i, m a x, s i m}$ the way it is outlined above. The first source of uncertainty is induced by the exponential fit itself. This uncertainty can be computed from the diagonal elements of the covariance matrix. The second one is due to the selection of the region where the exponential fit is applied. A too broad fitting region leads to an underestimation of the growth rates. On the other hand, if it is too narrow, only some local fluctuations of the exponential curve may be considered. We tested several fitting regions and analysed the anticipated uncertainties. For simplicity, we conservatively estimated the total uncertainty by multiplying the one from the fit by a factor of two. We note that this, as a matter of course, does not include uncertainties due to the numerical simulations themselves.

Another quantity to compare with linear theory is the fractional bandwidth $\Delta \omega_{\mathrm{r}} / \omega_{\mathrm{r}}$. This quantity cannot be directly estimated from the functional dependence of the frequency on the wavenumber in the simulations. This is because our simulations do not have enough time steps in the growing phase of the instability to sufficiently cover the frequency space. However, we can estimate the fractional bandwidths from our simulations in a different way: The fractional bandwidth is first estimated as the full width at half maximum (FWHM) from the growth rates as a function of the wavenumber, $\Delta k$, normalised to the wavenumber of the maximal growth rate $k\left(\omega_{i, \max }\right)$, that is to say $\Delta k / k\left(\omega_{\mathrm{i}, \max }\right)$. By assuming that the positive growth rates are present as waves that have a phase speed $v_{\phi} \approx c$, a linear relation between frequency and wavenumber holds. This allows us to map the wavenumber range into a frequency range, meaning

\footnotetext{
4 We note that the growth rate fit function does not have a factor of two in the exponent because we used the electric field amplitude, not the electric energy. For the same reason, we chose different fit constants $e_{0}, e_{1}$
} 
that $\Delta \omega_{\mathrm{r}} / \omega_{\mathrm{r}}=\Delta k / k$. To obtain an estimation of the uncertainties inherent in the calculation of this quantity, each fractional bandwidth value is calculated for two growth rate profiles as the FWHM of the functions $\omega_{\mathrm{i}}(k) \pm \omega_{\mathrm{i} \text {,err }}(k)$, where $\omega_{\mathrm{i} \text {,err }}(k)$ are the uncertainties of the growth rates estimated from the fit described above. The resulting fractional bandwidth is the mean of these two values of the FWHM, while the uncertainty of the fractional bandwidth corresponds to the standard deviation.

The enhanced PIC shot noise (compared to the real thermal fluctuations) due to the limited number of particles per Debye length in a cold plasma appears not to influence the instability evolution significantly. Instead, our results are not very sensitive to the temperature for the cold plasma limit $\rho \rightarrow \infty$ (Figs. 3 . 5). We tested the convergence of simulations 6 and 10 in order to cover a large range of temperatures, beam velocities, and numbers of particles per Debye length in this convergence study. In doing so, we either increased the number of particles by a factor of eight, or increased the domain size by a factor of ten, and re-estimated their integrated growth rates, growth rates, and fractional bandwidths. All of them are consistent with the values given in Table 1 within the estimated error interval.

\section{Results}

First of all, we assume an inverse temperature of $\rho_{0}=\rho_{1}=1$ for both beam and background plasma (corresponding to a temperature of $T \approx 10^{10} \mathrm{~K}$ ) and a beam streaming with a Lorentz factor of $\gamma_{\mathrm{b}} / \gamma_{\mathrm{b} \text {,min }}=2$, where $\gamma_{\mathrm{b}, \min }$ is the minimum value of $\gamma_{\mathrm{b}}$ at which the background and beam distributions are, according to Penrose's instability criterion of Eq. (7), just well enough separated. The latter criterion, however, is not valid for $\rho_{0}<1$ : In this case, the distributions of beam and background plasma show a significant overlap. We note that in all figures of this Section, frequencies are always normalised to the plasma frequency $\omega_{\mathrm{p}}$ while wavenumbers $k$ are given in units of the electron skin depth $d_{\mathrm{e}}=c / \omega_{\mathrm{p}}$.

Fig. 1 shows a comparison of a numerical solution of the linear dispersion relation with the results of the PIC Simulation 1, displaying both the real $\left(\omega_{\mathrm{r}}\right)$ and imaginary $\left(\omega_{\mathrm{i}}\right)$ parts of the frequency as a function of $k$ in the range where a streaming instability is excited. The real solution includes two dispersion branches: the superluminal L-mode branch, depicted by a dashdotted red line, and subluminal branch, represented as a dashed magenta line. Beyond that, the figure depicts the FFT $\boldsymbol{E}(k, \omega)$ of the power of the electric field oscillations $\boldsymbol{E}(x, t)$ obtained by our simulations. It represents the power spectral density (blue colour). This quantity was obtained by analysing the evolution of the electric field over 26000 equidistantly selected time steps in the time interval $(0-3250) \omega_{\text {pe }} t$. Thus, Fig. 1 a demonstrates that the regions in the $\omega-k$ diagram with the strongest power spectral density agree very well with the locations of the wave modes as predicted by the linear dispersion relation.

The growth rates of both approaches are compared for the subluminal branch in Fig. 1p. This is the wave mode to be further investigated in the following subsections. The maximum value of the growth rate $\omega_{\mathrm{i}} / \omega_{\mathrm{p}}=8.7 \times 10^{-4}$ occurs at a wavenumber of $k d_{\mathrm{e}} \approx 1.66$. The wing of the growth rate curve to the left of its peak value is slightly higher in the simulation than in the linear dispersion relation, while the right wing has almost the same shape in both approaches. The integrated growth rate computed analytically, shown as a horizontal red line, is in good agreement with the one from simulation (horizontal black line). In all our cases, the integrated growth rate $\Gamma$ is lower than or equal to the maximum growth rate $\omega_{i, \max }$. The former may only reach the maximum growth rate in those cases in which the $\omega_{\mathrm{i}}-k$ dependence features a very narrow peak. The parameters used in Fig. 1 will be kept constant in the following while separately changing a single parameter in order to assess its effects on instability growth and wave generation.

\subsection{Beam density}

To begin with, the question of the dependence of the wave growth on the beam-to-background density ratio is addressed. We vary the parameter $r_{\mathrm{n}}$ from very small values $\left(r_{\mathrm{n}}=10^{-4}\right.$, a weak beam) up to the scenario of a strong beam $\left(r_{\mathrm{n}}=1\right)$, while keeping the beam drift speed constant at $\gamma_{\mathrm{b}}=26$.

Fig. 2 illustrates the effect of altering the density ratio on the wave growth, showing the maximum as well as integrated growth rates and fractional bandwidths as a function of different density ratios $r_{\mathrm{n}}$. We note that for $r_{\mathrm{n}}=1$, the beam plasma has to be represented by 26000 (macro-) particles per cell, which means it contains 26 times more particles than the background. The maximum and integrated growth rates as well as the fractional bandwidths grow with increasing beam density, but not linearly. The agreement between simulation and analytical theory proves to be better in case of the integrated growth rates that follow the analytical trend. While the simulation maximum growth rates are very similar to the linear dispersion relation calculations for small $r_{\mathrm{n}}$, they tend to be lower than the analytical calculations for higher density ratios. The fractional bandwidths from simulations are in reasonable agreement with those from linear theory with a slight trend towards lower values than the analytical ones.

To predict the maximum growth rates that may occur for a certain density ratio, a fit function of the following form suits the observed dependence:

$f\left(r_{\mathrm{n}}\right)=a \cdot\left(r_{\mathrm{n}}+b\right)^{d}$,

where $a, b$, and $d$ are constants. If the growth rate is zero for a case without beam, the parameter $b$ should be zero. In our case, it approaches zero. The growth rate has a lower than linear dependence on the beam-to-background density ratio, which is reflected by a fit parameter $d<1$. The integrated growth rates and bandwidths follow the same behaviour as the maximum growth rates, such that they can be described a fit function of the same shape with different values for the parameters $a, b$ and $d$. A summary of these fit parameters is to be found in Table 2

\subsection{Beam temperature}

This subsection is dedicated to the results with regard to the influence of the (inverse) beam temperature $\rho_{1}$ on the growth rates $\omega_{\mathrm{i} ; \max }$ and $\Gamma$ as well as on the fractional bandwidths $\Delta \omega_{\mathrm{r}}$ at the frequency of maximum growth.

Fig. 3 shows the behaviour of these growth rates and bandwidths depending on the beam temperature, varying it between $\rho_{1}=1$ and $\rho_{1}=100$ in both analytical theory and simulation. For beam temperatures approaching the non-relativistic regime, the maximum growth rate firstly increases until it approaches a saturation level for the limit of a very cold beam, $\rho_{1} \rightarrow \infty$. A similar dependence can be found for the integrated growth rates and fractional bandwidths. A fit function of the following form describes this behaviour:

$f\left(\rho_{1}\right)=a-\frac{b}{\left(c+\rho_{1}\right)^{d}}$, 

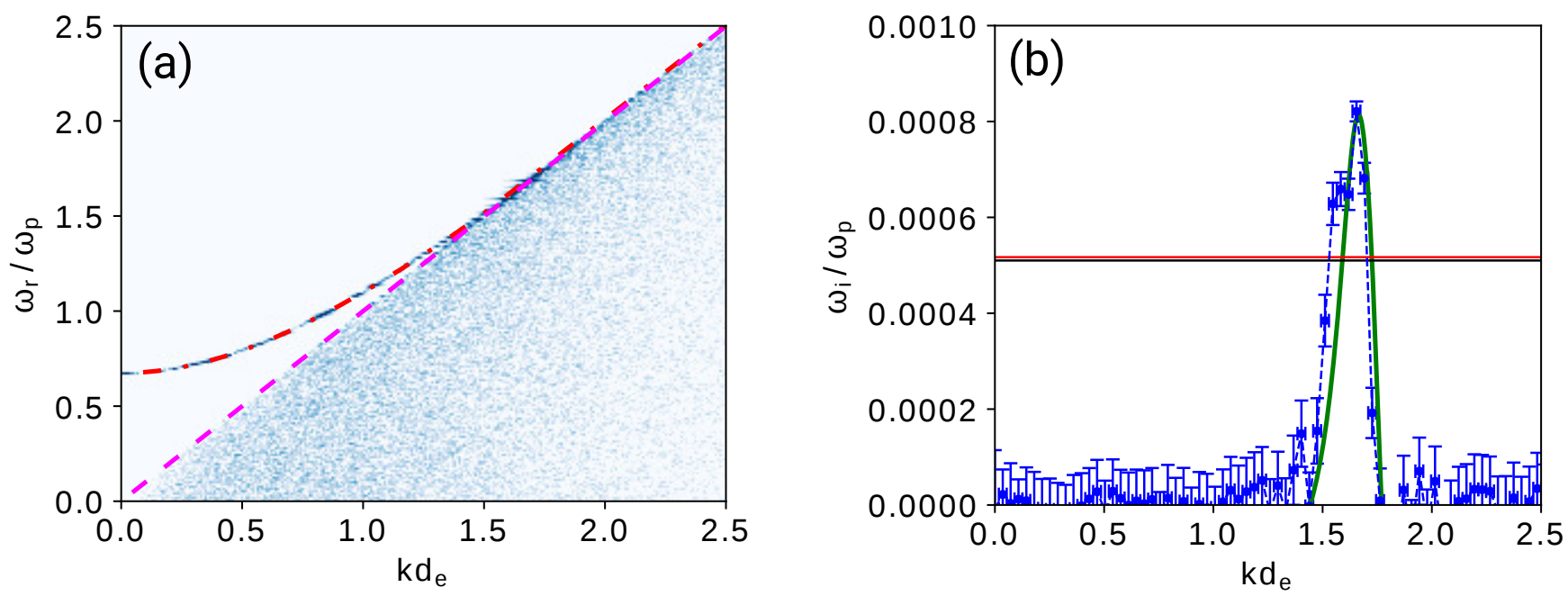

Fig. 1. Comparison between a solution obtained by linear theory with the one from simulation for $\rho_{0}=\rho_{1}=1, \gamma_{\mathrm{b}}=26, r_{\mathrm{n}}=10^{-3}$. (a) Dispersion diagram showing the real part of the frequency $\omega_{\mathrm{r}}$ as a function of the wavevector $k$. The background in blue colour scale represents the power spectral density obtained from the simulation electric field $\boldsymbol{E}(x, t)$. The discontinuous lines represent solutions of the linear dispersion relation Eq. 1 the superluminal L-mode branch (dash-dotted red line) and the subluminal branch (dashed magenta line). (b) Imaginary part of the frequency $\omega_{\mathrm{i}}$ (corresponding to the growth rates) as a function of the wavenumber $k$ for the subluminal branch. The green line shows the solution of the linear dispersion relation, and the dashed blue lines with error bars indicate the simulation results. The horizontal lines represent the values of the integrated growth rates obtained from the dispersion relation (red line) and from the simulation (black line).
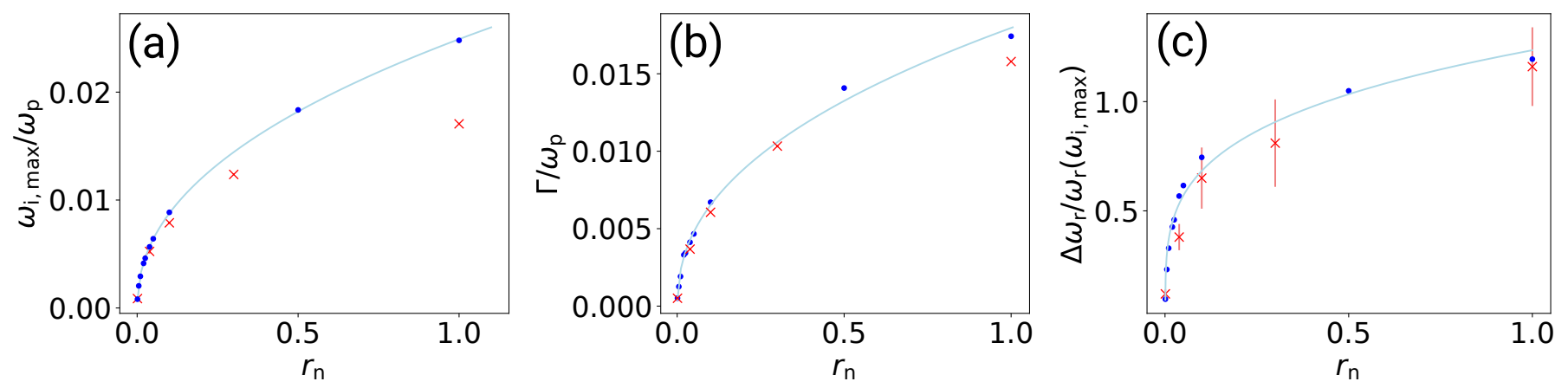

Fig. 2. Influence of the beam density on the wave growth and fractional bandwidths, investigated by varying the beam-to-background density ratio $r_{\mathrm{n}}$ from $10^{-4}$ to 1: (a) Maximum growth rates $\omega_{\mathrm{i}}$ for different beam-to-background density ratios $r_{\mathrm{n}}$ obtained by solving the linear dispersion relation (dots) and from simulations (crosses) with fit function (solid line). (b) Integrated growth rates calculated from numerical solutions of the dispersion relation (dots) and from simulation results (crosses) with fit function (solid line). (c) Fractional bandwidth $\Delta \omega_{\mathrm{r}}$ at the point where $\omega_{\mathrm{i}}$ is at its maximum, normalised to the corresponding real part of the frequency $\omega_{\mathrm{r}}$, obtained by linear theory (dots) and simulation (crosses). All three figures are for $\rho_{0}=\rho_{1}=1$ and $\gamma_{\mathrm{b}}=26$. Details regarding the fit functions can be found in Table 1

where the parameter $a$ corresponds to the saturation value that can be reached for the beam temperature approaching zero. This saturation occurs at $\rho_{1} \approx 18$. The parameters $b$ and $d$ determine how quickly the growth rate converges to this saturation value with decreasing temperature. This way, Eq. (16) quantifies the increase in growth of the instability with higher $\rho_{1}$ due to the increasing narrowness and steepness of the beam velocity distribution function. The specific values of the fit parameters are indicated in Table 2 .

Comparing the analytical results with our simulations, it can be stated that the maximum growth rates from simulation agree with the solutions of the linear dispersion relation very well, while the simulation integrated growth rates slightly exceed the analytical ones. In the case of the fractional bandwidths, the simulation values are below those obtained by linear theory. How- ever, they have large uncertainties that include the linear theory results in two of the three cases.

\subsection{Background temperature}

A similar investigation can be carried out by changing the temperature of the background plasma while keeping the beam temperature constant at $\rho_{1}=1$. The obtained maximum and integrated growth rates also obey the same behaviour as those in Sect. 4.2 and can thus be described by a fit function of the same form Eq. 16. These fit functions are displayed in Fig. 4, the parameters being stated in Table 2. Also in this case, saturation is observed at $\rho_{0} \approx 18$. However, a different behaviour is found for the fractional bandwidths: They decrease with lower background temperatures. With respect to the agreement between analytical theory and simulation, the same tendency as in Sect. 4.2 is ob- 

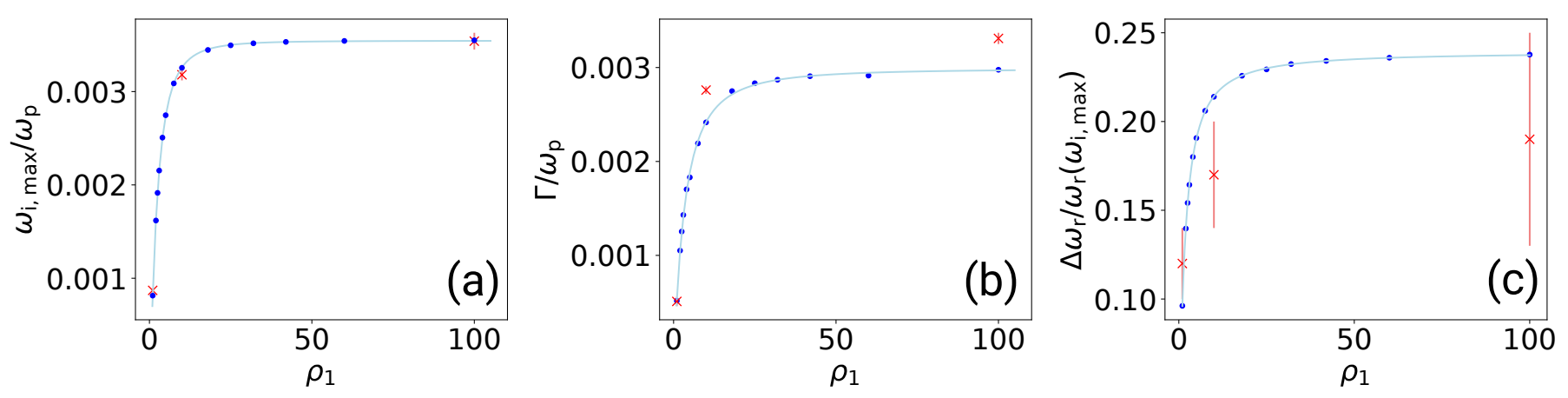

Fig. 3. Influence of the (inverse) beam temperature $\rho_{1}$ : (a) Maximum growth rates $\omega_{\mathrm{i}}$ for different beam inverse temperatures $\rho_{1}$ obtained from the analytical solution of the linear dispersion relation (dots) and from simulations (crosses) with fit function (solid line). (b) Integrated growth rates from solutions of the dispersion relation (dots) and from simulations (crosses) with fit function (solid line). (c) Fractional bandwidth $\Delta \omega_{\mathrm{r}}$ at the point where $\omega_{\mathrm{i}}$ is at its maximum, normalised to the corresponding real part of the frequency $\omega_{\mathrm{r}}$ obtained by linear theory (dots) and simulation (crosses). All three figures are for $\rho_{0}=1, \gamma_{\mathrm{b}}=26$ and $r_{\mathrm{n}}=10^{-3}$. All fit parameters are included in Table 1 .
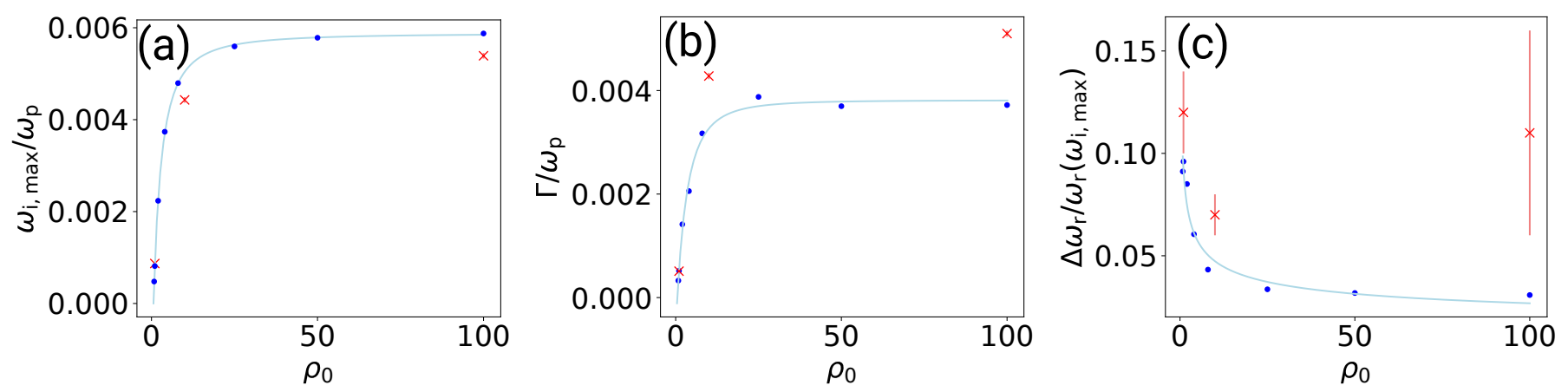

Fig. 4. Influence of the (inverse) background temperature $\rho_{0}$; same as Fig. 3, but varying $\rho_{0}$ instead of $\rho_{1}$ for $\rho_{1}=$ const. $=1$.

served in the case of the integrated growth rates, namely that the simulation results are larger than the linear theory values. Meanwhile the agreement is better for the maximum growth rates, with the simulation growth rates however being slightly lower than the theoretical predictions. In the case of the fractional bandwidths, however, the simulation results differ significantly from those obtained by linear theory. They do not decrease as much as predicted by the theoretical curve, more specifically, the simulation results are always higher. In addition, they actually follow the opposite trend as the analytical curve for a very cold background, meaning that the simulation results increase while the theoretical curve decreases.

\subsection{Background and beam temperature}

Since either the temperature of the beam or the background plasma is always kept at $\rho_{0 / 1}=1$ in the preceding Sects. 4.2 and 4.3 , it is worth to explore the behaviour for a different overall temperature. This means that we simultaneously change $\rho_{0}$ and $\rho_{1}$ while their ratio is set to $r_{\rho}=1$. This temperature ratio corresponds to the one considered in the work by Rafat et al. (2019a). The maximum and integrated growth rates as well as fractional bandwidths as a function of the overall temperature are shown in Fig. 5.

A similar explanation as in Sect. 4.2 applies to the increase in growth rates with decreasing temperature, that is to say it is due to the steepening of both beam and background velocity distribution functions. In this case, the growth rates are higher and the rise is steeper than in the cases where the temperature of only one component is decreased. A saturation of the maximum and integrated growth rates from the solutions of the linear dispersion relation occurs at approximately the same temperature of the beam or background, respectively, as observed in the previously investigated cases. However, the maximum and integrated growth rates from simulations - which have again slightly lower maximum and slightly higher integrated values - do not saturate after this value but rather start to decrease again.

The fractional bandwidth shows a different behaviour. After a steep rise until $\rho_{0} \approx 1.5$, it starts to decrease again. For values of $\rho_{0}<1.5$ the behaviour is governed by the beam temperature, for which the bandwidth rises when the beam gets colder, while the background temperature starts to dominate the behaviour for $\rho_{0}>1.5$. This decrease is also loosely observed in the simulation results. Although their values are always above the analytical ones and diverge significantly for the coldest temperature case, the error bars of the simulation results barely include the analytical curve. In this sense, the disagreement is not as severe as for Figure. 4. The different behaviour of the fractional bandwidths leads to the fact that the fit function $(16)$ is not sufficient to capture the dependence and has to be extended in the following way:

$f\left(\rho_{0 / 1}\right)=a-\frac{1}{\left(\rho_{0 / 1}+b\right)^{c}}+\frac{d}{\left(\rho_{0 / 1}\right)^{e}}$.

The specific values of the fit parameters for the curves depicted in Fig. 5are given in Table 2

\subsection{Beam drift speed for constant $r_{\mathrm{n}}$}

In the following, we analyse the effects of the beam drift speed on the growth rates. We note that changing the beam drift speed 

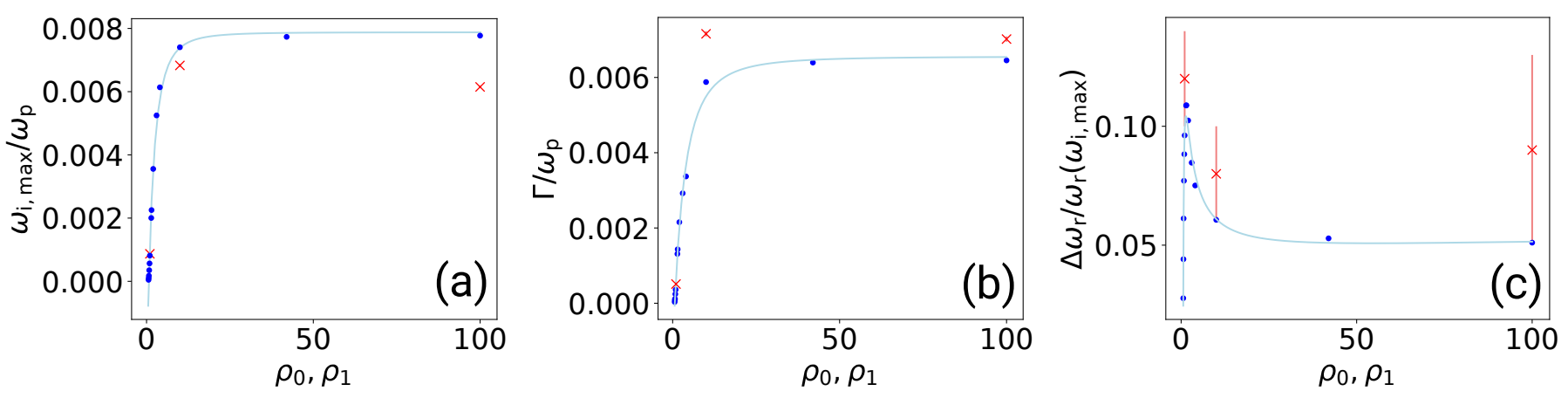

Fig. 5. Influence of the (inverse) overall (beam and background) temperature $\left(\rho_{0}=\rho_{1}\right.$, corresponding to $r_{\rho}=1$ ); same as Fig. 3 . but simultaneously changing $\rho_{0}$ and $\rho_{1}$ instead of solely $\rho_{1}$.

under the assumption of a weak beam with $r_{\mathrm{n}}=10^{-3}$ necessarily implies a change of the particle density ratio $n_{1} / n_{2}$ because of the dependence of $r_{\mathrm{n}}$ on the beam Lorentz factor.

As it can be inferred from Fig. 6, firstly, the growth rates, the integrated growth rates as well as the fractional bandwidths increase with faster beam velocities until they reach a maximum value for $\gamma_{\mathrm{b}} \approx 75$. Subsequently, they decrease with increasing beam velocity. The extension of the fit function (16), given by Eq. 17, can also be applied here to describe the dependence of the growth rates as well as bandwidths on $\gamma_{\mathrm{b}}$. The second term of Eq. (17) is connected with the increase in growth rates for $\gamma_{\mathrm{b}}<75$ while the third term describes the growth rate decrease for $\gamma_{\mathrm{b}}>75$. The corresponding parameters of the fitted curves are given in Table 2 . In the case of the simulational growth rates, it cannot be clearly stated whether they follow the exact same behaviour as the analytical ones since they have not, so far, reached the regime where they would decrease again. A reasonable agreement, however, is found for the integrated growth rates at lower $\gamma_{\mathrm{b}}$, while the value of $\Gamma$ is higher than the analytical solution for a higher $\gamma_{\mathrm{b}}$. The maximum growth rates follow the trend that is observed for the change of all investigated parameters as they tend to be lower than the analytically calculated ones. Equally, the behaviour of the fractional bandwidths prevents a clear conclusion towards the agreement with linear theory as it appears to rather be opposed to the analytical curve.

\subsection{Beam drift speed for $n_{0}=n_{1}$}

Within the Usov (1987) model, it is sensible to assume approximately equal densities of the beam and background plasma. Fig. 7] shows the maximum and integrated growth rates and the fractional bandwidths for increasing beam velocities while keeping the ratio of particle number densities $n_{0}$ and $n_{1}$ constant.

As in the case analysed in the preceding Sect. 4.5, the growth rates increase with higher beam velocity, this time up to a value of $\gamma_{\mathrm{b}} \approx 30$. After reaching the maximum, the growth rates subsequently decrease. Compared to Sect. 4.5, however, this decrease is at first steeper with increasing $\gamma_{\mathrm{b}}$ until it levels off at large $\gamma_{\mathrm{b}}$. The extended fit function (17) is suited to describe the behaviour of the growth rates here as well, assuming the fit parameters summarised in the Table 2. By contrast, the fractional bandwidths exhibit a different behaviour than in the case analysed before: They monotonously decrease for the entire beam velocity range. They can be described by the function $(16)$ with fit parameters given in Table 2. The simulational and analytical growth rates match well for this case. Furthermore, the fractional bandwidths from simulations follow the same trend as those obtained by lin- ear theory, although the curve indicated by the simulation points might be slightly flatter than the analytical one.

\section{Discussion}

To study one of the possible radio emission mechanisms of pulsars, we used a model based on the mutual interaction of two relativistic beams (Usov 1987). We investigated the properties of this kinetic instability for a wide range of plasma parameters, using an appropriate one-dimensional Maxwell-Jüttner velocity distribution. As an initial validation test, we compared our linear dispersion results with those by Rafat et al. (2019b) and with PIC simulations results (see Fig. 1 as an example). We found only some small discrepancies between both approaches in the whole investigated parameter range, mostly caused by non-linear effects present in PIC simulations. Beyond the analytical results, based on the linearisation of the Vlasov equation, our simulations also include the saturation of the instability after the linear growing phase, when the exponential wave growth becomes too large for the linear approximation to be valid. Once the wave amplitude stops to grow, the system is dominated by those nonlinear effects that might be responsible for the observed differences. With both approaches, we obtained positive growth rates for the entire investigated parameter range.

Now the question arises whether this instability can account for the observed strong pulsar radio emission. According to Ursov \& Usov (1988), the time for instability development must be at least one order smaller than the typical lifetime of the system - the time span between two plasma bunch releases. This time span until the next bunch release, $\Delta t$, can be estimated to be of the order of $10 \mu$ s (Ruderman \& Sutherland 1975, Asseo \& Melikidze 1998). Thus, in order to quantify the effectiveness of the instability, it is possible to estimate the growth time of the instability assuming a growth factor of $G=1$ (Eq. (9)), which means at the transition between efficient and inefficient wave growth. For this, we compute $\omega_{\mathrm{i}}$ for this case by taking the inverse of the imaginary part of the obtained frequency. If $\Delta t=G / \omega_{\mathrm{i}}<10 \mu \mathrm{s}$, the instability can be considered effective. The plasma frequency can be estimated as $\omega_{\mathrm{p}} \approx 3.6 \times 10^{9} \mathrm{~s}^{-1}$ (Ursov \& Usov 1988) at the distance from the pulsar where the instability is expected to develop. We assume that the normalised growth rate value $\omega_{\mathrm{i}} / \omega_{\mathrm{p}}$ is invariant under Lorentz transformations. Then the time interval $\Delta t=10 \mu \mathrm{s}$ determines a threshold for the minimum growth rate in the pulsar frame: $\omega_{\mathrm{i}, \text { thr }} / \omega_{\mathrm{p}}=1 /\left(\Delta t \omega_{\mathrm{p}}\right)=2.8 \times 10^{-4}$. Thus, according to this criterion, all unstable waves with growth rates $\omega_{\mathrm{i}} / \omega_{\mathrm{p}}>\omega_{\mathrm{i}, \text { thr }} / \omega_{\mathrm{p}}$ would have sufficient time to develop during the mutual bunch interactions. 

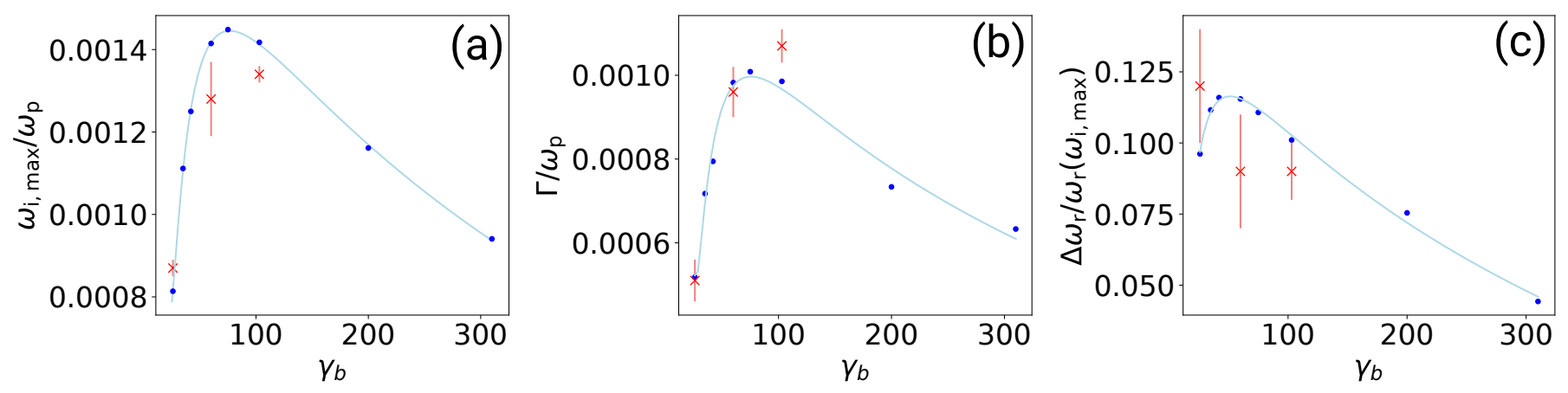

Fig. 6. Influence of the beam velocity in the weak-beam limit $n_{1} \ll n_{0}$ (i.e. $r_{\mathrm{n}}=10^{-3}$ ). (a) Maximum values of $\omega_{\mathrm{i}}$ for different beam velocities $\gamma_{\mathrm{b}}=\left(1-\beta_{\mathrm{b}}^{2}\right)^{-\frac{1}{2}}$ obtained from the solutions of the linear dispersion relation (dots) and simulations (crosses) with fit function (solid line). (b) Integrated growth rates from numerical solutions of the dispersion relation (dots) and from simulations (crosses) with fit function (solid line). (c) Fractional bandwidth $\Delta \omega_{\mathrm{r}}$ at the point where $\omega_{\mathrm{i}}$ is at its maximum, normalised to the corresponding real part of frequency $\omega_{\mathrm{r}}$, obtained from linear theory (dots) and simulation (crosses). All three figures for $\rho_{0}=\rho_{1}=1, r_{\mathrm{n}}=10^{-3}$.
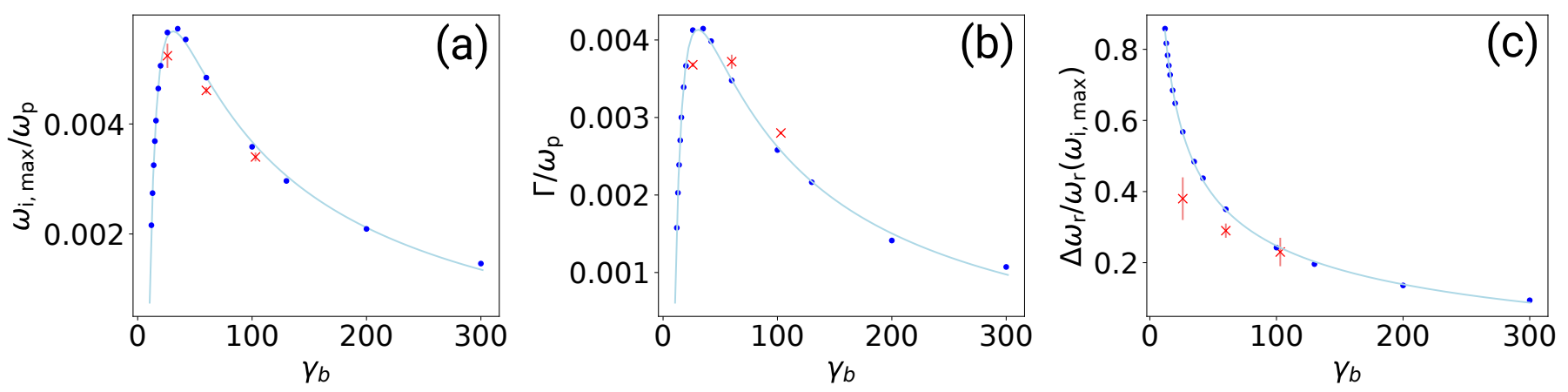

Fig. 7. Influence of the beam velocity $\gamma_{\mathrm{b}}$ in the case of equal beam and background densities $\left(n_{1}=n_{0}\right)$; Same as Fig. 6 , but for a density ratio of $n_{0}=n_{1}$.

We note, however, that this threshold growth rate depends on the underlying model. For example, Weatherall (1994) states higher values for the typical pulsar plasma density of $n_{\mathrm{e}} \approx$ $9.7 \cdot 10^{13} \mathrm{~cm}^{-3}$. The corresponding plasma frequency $\omega_{\mathrm{p}} \approx$ $7.9 \cdot 10^{11} s^{-1}$ would lead to a smaller threshold growth rate of $\omega_{\mathrm{i}, \mathrm{thr}} / \omega_{\mathrm{p}}=1.3 \times 10^{-6}$, providing an even broader parameter range for a sufficiently strong instability. However, the model by Weatherall (1994) assumes a strong-beam model with counterstreaming electron and positron populations as a major source of the instability that causes the coherent pulsar radio emission. Thus, the threshold given in the preceding paragraph appears to be better applicable to our investigated case and presupposed model.

Comparing this threshold value of $\omega_{\mathrm{i}, \text { thr,pulsar }} / \omega_{\mathrm{p}}=2.8 \times 10^{-4}$ with our analytical and simulation results for all analysed parameters, the instability appears to be strong enough to develop - and may thus lead to the generation of significant radio emission for density ratios $r_{\mathrm{n}}>10^{-3}$ and for the beam and background inverse temperatures $\rho_{0}, \rho_{1} \geq 1$. For small $\rho_{0}, \rho_{1}<1$, however, the distribution functions of of both beam and background overlap significantly. This temperature range is in agreement with Weatherall (1994). Beyond that, the growth rates exceed the threshold for beam velocity Lorentz factors $13<\gamma_{\mathrm{b}}<300$. The beam velocity must be large enough to separate both distributions while the temperature has to be low enough in order to guarantee that the relativistic broadening of the distribution does not quench the instability. We note that all our simulations have a growth rate that is high enough to overcome the growth rate threshold.
Contextualising the findings of our parametric study within the existing models, we can bridge the gap between those models assuming a weak beam and those focusing on a strong-beam instability. We show how the growth rates and the fractional bandwidths increase with a higher beam density. This implies that the velocity distribution slope increases, such that the instability becomes stronger. This dependence is not linear. Since for larger $r_{\mathrm{n}}$, a deviation between the analytical and simulation growth rate is observed, the extent of the increase in the achievable growth rates with larger beam density is not clear. Nevertheless, we can conclude that an increase in $r_{\mathrm{n}}$ to $\sim 0.3-$ which is the point where both simulation and analytical growth rates still agree with each other - significantly enhances the instability growth rates. While the model by Rafat et al. (2019b) explicitly assumes the weak-beam condition, $r_{\mathrm{n}} \ll 1$, the theory discussed in Usov (1987) is not limited to just a weak beam. A rather strong-beam model would in principle be conceivable within the Usov (1987) model since both successively emitted bunches initially feature equal densities, and the relative velocity between the plasma that forms the beam and the one that can be considered the background might be not as large as initially suggested by Usov (1987).

We also study the transition between hot and cold beam and background distributions. With decreasing temperature, a colder distribution leads to a larger slope of the corresponding velocity distribution function, causing a stronger instability. As analysed in Sect. 4.2 for low values of the inverse beam temperature $\left(\rho_{1} \approx 1\right)$, the distribution function is very broad and the growth rates are dominated by relativistic effects. With increas- 
Table 2. Parameters of the fitted functions for the growth rates, the integrated growth rates, and the fractional bandwidths.

\begin{tabular}{|c|c|c|c|c|c|c|}
\hline Figure & Equation & $a$ & $b$ & $c$ & $d$ & $e$ \\
\hline \multicolumn{7}{|c|}{ Growth rates } \\
\hline 2 (a) & Eq. 15 & $(2.491 \pm 0.009) \times 10^{-2}$ & $(-6 \pm 1) \times 10^{-4}$ & - & $(4.51 \pm 0.03) \times 10^{-1}$ & - \\
\hline$\sqrt{3}$ a) & Eq. 16 & $(3.544 \pm 0.004) \times 10^{-3}$ & $1.0 \pm 0.4$ & $6.6 \pm 0.4$ & $2.9 \pm 0.1$ & - \\
\hline (4) & Eq. $\overline{16}$ & $(5.88 \pm 0.37) \times 10^{-3}$ & $(5 \pm 3) \times 10^{-2}$ & $3.3 \pm 0.6$ & $1.6 \pm 0.2$ & - \\
\hline 5 (a) & Eq. 16 & $(7.9 \pm 0.2) \times 10^{-3}$ & $1.0 \pm 0.0$ & $4.9 \pm 0.3$ & $2.81 \pm 0.08$ & - \\
\hline (6) & Eq. 17 & $-19 \pm 1$ & $(-6.7 \pm 0.6) \times 10^{-4}$ & $(-1.8 \pm 0.6) \times 10^{-1}$ & $1.3 \pm 0.1$ & $1.0049 \pm 0.0004$ \\
\hline 7 (a) & Eq. 17 & $2.1 \pm 0.4$ & $1 \pm 6$ & $0.3 \pm 7$ & $0.7 \pm 4$ & $(-2 \pm 6) \times 10^{-3}$ \\
\hline \multicolumn{7}{|c|}{ Integrated growth rates } \\
\hline $2(b)$ & Eq. 15 & $(1.80 \pm 0.04) \times 10^{-2}$ & $(-8 \pm 5) \times 10^{-4}$ & - & $(4.4 \pm 0.2) \times 10^{-1}$ & - \\
\hline 3 (b) & Eq. 16 & $(2.99 \pm 0.03) \times 10^{-3}$ & $(9 \pm 8) \times 10^{-2}$ & $6 \pm 1$ & $1.8 \pm 0.3$ & - \\
\hline $4(\mathrm{a})$ & Eq. 16 & $(3.81 \pm 0.9) \times 10^{-3}$ & $1 \pm 0$ & $8.1 \pm 0.7$ & $2.6 \pm 0.1$ & - \\
\hline 5 (b) & Eq. $1 \overline{16}$ & $(6.6 \pm 0.1) \times 10^{-3}$ & $1.0 \pm 0.0$ & $7.6 \pm 0.4$ & $2.39 \pm 0.05$ & - \\
\hline 6(b) & Eq. $\overline{17}$ & $-23 \pm 3$ & $(-4 \pm 2) \times 10^{-4}$ & $(-4 \pm 8) \times 10^{-1}$ & $(1.6 \pm 0.6) \times 10^{-1}$ & $1.003 \pm 0.001$ \\
\hline 7 (b) & Eq. $\overline{17}$ & $2 \pm 71$ & $1 \pm 10$ & $(3 \pm 1) \times 10^{-1}$ & $(8 \pm 6) \times 10^{-1}$ & $(-7 \pm 0.4) \times 10^{-4}$ \\
\hline \multicolumn{7}{|c|}{ Fractional bandwidths } \\
\hline $2(c)$ & Eq. 16 & $1.24 \pm 0.04$ & $(-9.6 \pm 0.0) \times 10^{-4}$ & - & $(2.6 \pm 0.1) \times 10^{-1}$ & - \\
\hline $3(\mathrm{~B})$ & Eq. $\overline{16}$ & $0.2392 \pm 0.0003$ & $0.51 \pm 0.03$ & $1.83 \pm 0.09$ & $1.22 \pm 0.03$ & - \\
\hline 武(c) & Eq. 16 & $0.08 \pm 0.02$ & $1 \pm 0$ & $0.3 \pm 0.2$ & $0.009 \pm 0.022$ & - \\
\hline $5(\mathrm{~s})$ & Eq. $\overline{17}$ & $-0.2 \pm 0.1$ & $0.5 \pm 0.3$ & $1.14 \pm 0.34$ & $0.52 \pm 0.06$ & $0.07 \pm 0.06$ \\
\hline 6(c) & Eq. $\overline{17}$ & $(-1.6 \pm 0.4) \times 10^{-2}$ & $(-6 \pm 1) \times 10^{-2}$ & $(-3 \pm 2)$ & $(9 \pm 7) \times 10^{-1}$ & $1.4 \pm 0.1$ \\
\hline $7(\mathrm{c})$ & Eq. 16 & $-0.23 \pm 0.06$ & $-2.6 \pm 0.3$ & $-1 \pm 1.3$ & $0.37 \pm 0.05$ & - \\
\hline
\end{tabular}

ing $\rho_{1}$, the growth rates approach a saturation $\left(\rho_{1}>18\right)$, even though the distribution still significantly deviates from a nonrelativistic Maxwellian distribution. The saturation values are consistent with the behaviour of the growth rates in reactive fluid instabilities within the cold beam approximation (Hinata 1976, Shalaby et al.2017):

$$
\frac{\omega_{\mathrm{i}}}{\omega_{\mathrm{p}}}=\frac{1}{2 \sqrt{2 \gamma_{\mathrm{b}}^{3}}} .
$$

For $\gamma_{\mathrm{b}}=26$, this expression yields $\omega_{\mathrm{i}} / \omega_{\mathrm{p}}=2.67 \times 10^{-3}$ From the fit (Table 2, parameter $a$ ), we can estimate the saturation of our analytically calculated maximum growth rates at $\omega_{\mathrm{i}} / \omega_{\mathrm{p}}=(3.542 \pm 0.004) \times 10^{-3}$ (for $\left.\rho_{1} \rightarrow \infty\right)$. The simulations for $\rho_{1}=100$ also lead to growth rates near this saturation value $\left(\omega_{\mathrm{i}} / \omega_{\mathrm{p}}=(3.54 \pm 0.08) \times 10^{-3}\right)$. The integrated growth rates experience a saturation at $\Gamma / \omega_{\mathrm{p}}=(2.98 \pm 0.04) \times 10^{-3}$ in the linear calculations and $\Gamma / \omega_{\mathrm{p}}=(3.31 \pm 0.06) \times 10^{-3}$ in the simulations. In the case of lowering the background temperature, the saturation value is higher than in the case of decreasing only the beam temperature. Due to the plasma bunch interaction, the behaviour of the growth rates when simultaneously changing the temperature of the background and the beam is expected. The resulting growth rates cannot be easily estimated by a simple interpolation from the growth rate dependence on either the beam or background temperature. The growth rate saturation value when varying both temperatures is higher than in the case of solely changing one temperature. Therefore, we expect that for the generation of strong emission, a colder temperature of both beam and background than predicted by the current theoretical models could occur, for example, if both interacting bunches have already cooled down when moving away from the pulsar.
Moreover, we analyse the growth rates as a function of the beam velocity for two cases. The first case is for constant $r_{\mathrm{n}}=\frac{n_{1}}{\gamma_{\mathrm{b}} n_{0}}$, while the second one is for a constant particle density ratio $n_{0} / n_{1}=1$. Throughout the investigated parameter range, the latter case always exhibits higher growth rates compared to the first one due to the higher beam density in the background reference frame. In both cases, the growth rates first increase with increasing beam velocity while the distributions are overlapping, until the velocity is sufficiently high for the velocity distributions to fully separate. The maximum growth rate as a function of the beam velocity is reached for lower beam velocities in the case of $n_{0}=n_{1}$ than for the case of $r_{\mathrm{n}}=10^{-3}$. This is because for the former case, $r_{\mathrm{n}}$ decreases with increasing beam velocity, thus lowering the growth rates. In the case $r_{\mathrm{n}}=10^{-3}$, the density ratio in the background frame remains constant, and the instability growth rates are not significantly quenched for higher beam velocities due to relativistic effects, that is to say due to a strong broadening of the beam distribution with increasing beam velocity. The case $n_{1}=n_{2}$, by contrast, represents a closed plasma system where the number of particles of both populations remains constant and only the beam velocity varies. Given the estimated range of Lorentz factors $\gamma$ of the respective bunches as stated in the introduction $\left(\gamma_{\min } \sim 10\right.$ to $\gamma_{\max } \sim 10^{3}-10^{4}$, see Sect 1 , our results suggest that the interaction responsible for the pulsar emission may not take place between the fastest particles from the successive bunch $\left(\gamma_{\max }\right)$ and the slowest ones from the one emitted before $\left(\gamma_{\min }\right)$, as initially suggested in the work of Usov (1987). Instead, the interaction between sets of particles with a not so large difference between their velocities might be crucial for the generation of the coherent pulsar radio emission. Another possibility would be that the essential interaction between the bunches takes place when the previously emitted bunch has already slowed down more than the successive one. This would imply that the difference between the minimum and maximum 
velocity is not as large anymore as it was right after their release. However, no known studies about a possible slow-down of the bunches are available so far.

Apart from the numerical predictions of the growth rates, all our analytical calculations are accompanied by PIC simulations that cover the same parameter space. The position of the dispersion branches is almost identical for all conducted simulations and the growth rate profiles have maxima for similar values of the wavenumber $k$. The fits carried out in case of the simulation growth rates feature a high precision for low density ratios and high temperatures, and they are reasonably accurate throughout the entire beam velocity range. The agreement between simulation and analytical solutions corresponds to a co-validation of both approaches. Yet, they deviate with decreasing temperature and increasing beam particle density.

The differences between PIC simulations, which solve the Vlasov-Maxwell system of equations, and linear theory calculations based on a linearisation of the same system were interpreted by Skoutnev et al. (2019) as being due to the enhanced PIC noise that might make the beams hotter than the assumed values, in this way weakening the linear theory predictions for the growth rates. This effect could be especially significant for cold temperatures since the ratio between the enhanced (due to the noise) thermal fluctuations and beam temperature is large. Yet, this effect does not equally apply to the strongly magnetised plasma described by one-dimensional velocity distributions in our case. Instead, the instability evolution, as it is quantified by the growth rates, is not significantly influenced by the temperature uncertainty as the growth rates are almost independent of the temperature for the cold plasma limit, $\rho \rightarrow \infty$. In addition, the growth rates are actually larger for simulations than the linear theory predictions. This conclusion was also confirmed by two test simulations, in which we increased either the number of particles per cell by a factor of eight or the domain size by factor of ten. The resulting quantities fit within the corresponding uncertainty intervals as estimated in Table 1 .

A different explanation of the observed deviations might apply to our case: As the differences between linear theory and simulations are found to be at large beam densities or lower temperatures, they might be caused by the different nature of the instability itself and the approximations that are considered in linear theory. Indeed, streaming instabilities for large beam densities or lower temperatures are usually within the regime known as reactive instabilities, characterised by a large wavenumber range and large growth rates (Melrose 1986). By contrast, high temperatures and lower beam densities favour the so-called kinetic or resonant instabilities that feature a narrower unstable bandwidth (around the resonant phase speeds) and smaller growth rates. We note that both are just different limits of the same instability. Specifically, this explanation would be consistent with the larger growth rates and fractional bandwidths found in the simulations in Figures 45. Assuming that our simulation results are not significantly influenced by numerical effects, and in this way closer to reality than linear theory, we can estimate the origin of the deviations found in the linear theory values to be higher-order effects not taken into account, in particular near the end of the linear stage of the instability evolution. Since reactive instabilities for large beam densities or lower temperatures have higher growth rates and more wave modes are excited (large bandwidth) than in the opposite regime, non-linear effects could modify the linear theory predictions due to possible large-amplitude waves partially captured during the linear fitting calculation in simulations. In other words, there could be a quicker breakdown of the linear theory predictions in the reactive instability limit than in the kinetic or resonant instability limit. However, further investiagtions building on this are required in order to find out the exact cause of the disagreement between linear theory and simulations in this parameter regime.

\section{Conclusions}

We investigated one of the promising pulsar radio emission mechanisms that is based on the release of plasma bunches by computing the growth rates as a solution of the relativistic kinetic dispersion relation and by fully kinetic PIC simulations. To this end, we carried out a parametric study, analysing the influence of the density ratio between beam and background, the beam and background temperatures, and the beam velocity. While some studies (Melrose \& Rafat 2017, Rafat et al. 2019b) show that the growth rates from such an interaction are not high enough to consider this instability as a viable radio emission mechanism, we prove that for a specific parameter range, sufficiently large growth rates can be achieved. For the instability to be efficient in the sense of being responsible for the pulsar radio emission generation, we found that the density ratio should be in the range $r_{\mathrm{n}} \geq 10^{-3}$, the inverse temperatures $\rho_{0}, \rho_{1}>1$, and the beam Lorentz factor $13<\gamma_{\mathrm{b}}<300$.

Moreover, we found a considerably good agreement between the analytical calculations (solutions of the linear dispersion relation) and PIC simulations. This was illustrated by a comparison between the growth rate profile as a function of the wave number. In both cases, the growth rate maxima are very similar and have the same location in the $k$-space. We showed that the growth rates approach the expected growth rate values from the cold reactive beam instability in both cases. There are, however, small differences, mostly in the cold plasma limit: The simulations show slightly larger growth rates than those of the linear theory predictions. This could be attributed to the quicker breakdown of linear theory in this parameter regime.

We also studied the transition between the regimes of a weak and strong beam by investigating the dependence on its density ratio with respect to the background population, and the growth rate saturation as a function of the background and beam temperature.

Based on our findings, the two-stream instability should be further investigated for our selected parameters as a possible cause for pulsar radio emission. In particular, a comparison with observations is conceivable. A possible comparable quantity might consist in radio emission fluxes that can be obtained from the given calculated quantities.

Acknowledgements. We gratefully acknowledge the developers of the ACRONYM code (Verein zur Förderung kinetischer Plasmasimulationen e.V.), and the financial support by the German Science Foundation (DFG) via the projects MU-4255/1-1 and BU-777-17-1 as well as by the Czech Science Foundation (GACR) via the project 20-09922J. This work was supported by The Ministry of Education, Youth and Sports from the Large Infrastructures for Research, Experimental Development and Innovations project "e-Infrastructure CZ - LM2018140". Part of the simulations were carried out on the HPC-Cluster of the Institute for Mathematics at the TU Berlin. Computational resources were also supplied by the project "e-Infrastruktura CZ" (e-INFRA LM2018140) provided within the program Projects of Large Research, Development and Innovations Infrastructures. The authors gratefully acknowledge the Gauss Centre for Supercomputing e.V. (WwW.gauss-centre.eu) for funding this project by providing computing time on the GCS Supercomputer SuperMUC-NG at Leibniz Supercomputing Centre (www.lrz.de), through the project pr27ta. We thank the referee for his/her valuable comments to improve this paper.

The data sets used to reproduce the plots of this paper can be found at Zenodo: https://zenodo.org/record/4049883\#.X65Xy-1CdPY 


\section{References}

Arendt, P. N. \& Eilek, J. A. 2002, Astrophys. J., 581, 451

Arons, J. 1981, Astrophys. J., 248, 1099

Asseo, E. \& Melikidze, G. I. 1998, Mon. Not. R. Astron. Soc., 301, 59

Baumjohann, W. \& Treumann, R. 1997, Basic Space Plasma Physics (Imperial College Press)

Beskin, V. S. 2018, Uspekhi Fiz. Nauk, 188, 377

Beskin, V. S., Gurevich, S. V., \& Istomin, Y. N. 1993, Physics of the pulsar magnetosphere (Cambridge University Press)

Blaskiewicz, M., Cordes, J. M., \& Wasserman, I. 1991, The Astrophysical Journal, 370, 643

Bret, A., Gremillet, L., Benisti, D., \& Lefebvre, E. 2008, Physical Review Letters, 100, 205008

Bret, A., Gremillet, L., \& Dieckmann, M. E. 2010, Physics of Plasmas, 17, 120501

Buschauer, R. \& Benford, G. 1977, Mon. Not. R. Astron. Soc., 179, 99

Cheng, A. F. \& Ruderman, M. A. 1977a, Astrophys. J., 212, 800

Cheng, A. F. \& Ruderman, M. A. 1977b, Astrophys. J., 214, 598

Cole, J. B. 1997, IEEE Transactions on Microwave Theory and Techniques, 45, 991

Cottrill, L. A., Langdon, A. B., Lasinski, B. F., et al. 2008, Physics of Plasmas, 15,082108

Diver, D. A. \& Laing, E. W. 2015, Physica Scripta, 90, 025602

D’Angelo, M., Fedeli, L., Sgattoni, A., Pegoraro, F., \& Macchi, A. 2015, Monthly Notices of the Royal Astronomical Society, 451, 3460-3467

Egorenkov, V. D., Lominadze, D. G., \& Mamradze, P. G. 1984, Astrophysics (Engl. Transl.), 19, 426

Eilek, J. \& Hankins, T. 2016, J. Plasma Phys., 82, 635820302

Esirkepov, T. 2001, Computer Physics Communications, 135, 144

Gedalin, M., Melrose, D. B., \& Gruman, E. 1998, Phys. Rev. E, 57, 3399

Godfrey, B. B., Newberger, B. S., \& Taggart, K. A. 1975, IEEE Trans. Plasma Sci., 3, 60

Goldreich, P. \& Julian, W. H. 1969, Astrophysical Journal, 157, 869

Hankins, T. H. \& Eilek, J. A. 2007, Astrophys. J., 670, 693

Hewish, A., Bell, S. J., Pilkington, J. D. H., Scott, P. F., \& Collins, R. A. 1968, Nature, 217, 709

Hinata, S. 1976, 44, 389

Jüttner, F. 1911, Ann. Phys., 339, 856

Kilian, P., Burkart, T., \& Spanier, F. 2012, in High Perform. Comput. Sci. Eng. '11, ed. W. E. Nagel, D. B. Kröner, \& M. M. Resch (Berlin, Heidelberg: Springer Berlin Heidelberg), 5-13

Kramer, M., Johnston, S., \& van Straten, W. 2002, Monthly Notices of the Royal Astronomical Society, 334, 523

Kärkkäinen, M., Gjonaj, E., Lau, T., \& Weiland, T. 2006, Proc. International Computational Accelerator Physics Conference

Lesch, H., Jessner, A., Kramer, M., \& Kunzl, T. 1998, Astronomy and Astrophysics, 332

Levenberg, K. 1944, 11, 164

Liu, K., Young, A., Wharton, R., et al. 2019, The Astrophysical Journal Letters, $885, \mathrm{~L} 10$

Lu, Y., Kilian, P., Guo, F., Li, H., \& Liang, E. 2020, Journal of Computational Physics, 413, 109388

Luo, Q. \& Melrose, D. B. 2001, Mon. Not. R. Astron. Soc., 325, 187

López, R. A., Muñoz, V., Viñas, A. F., \& Valdivia, J. A. 2014, Physics of Plasmas, 21, 032102

López, R. A., Navarro, R. E., Moya, P. S., et al. 2015, The Astrophysical Journal, 810,103

Marquardt, D. W. 1963, Journal of the Society for Industrial and Applied Mathematics, 11, 431-441

Melrose, D. B. 1978, The Astrophysical Journal, 225, 557

Melrose, D. B. 1986, Instabilities in Space and Laboratory Plasmas (Cambridge: Cambridge University Press)

Melrose, D. B. 2017, Reviews of Modern Plasma Physics, 1, 5

Melrose, D. B. \& Gedalin, M. E. 1999, Astrophys. J., 521, 351

Melrose, D. B. \& Rafat, M. Z. 2017, in IOP Conf. Ser. 932, 012011

Petrova, S. A. 2009, Monthly Notices of the Royal Astronomical Society, 395, 1723

Philippov, A., Timokhin, A., \& Spitkovsky, A. 2020, Phys. Rev. Lett., 124, 245101

Powell, M. J. D. 1964, The Computer Journal, 7, 155

Rafat, M. Z., Melrose, D. B., \& Mastrano, A. 2019a, J. Plasma Phys., 85, 905850305

Rafat, M. Z., Melrose, D. B., \& Mastrano, A. 2019b, J. Plasma Phys., 85, 905850603

Rahaman, S. M., Mitra, D., \& Melikidze, G. I. 2020, Monthly Notices of the Royal Astronomical Society, 497, 3953

Ruderman, M. A. \& Sutherland, P. G. 1975, Astrophys. J., 196, 51

Shalaby, M., Broderick, A. E., Chang, P., et al. 2017, The Astrophysical Journal, 841,52
Shalaby, M., Broderick, A. E., Chang, P., et al. 2018, The Astrophysical Journal, 859,45

Shukla, C., Das, A., \& Patel, K. 2015, Physics of Plasmas, 22, 112118

Silva, L. O., Fonseca, R. A., Tonge, J. W., et al. 2003, The Astrophysical Journal, 596, L121-L124

Skoutnev, V., Hakim, A., Juno, J., \& TenBarge, J. M. 2019, The Astrophysical Journal, 872, L28

Stenson, E. V., Horn-Stanja, J., Stoneking, M. R., \& Pedersen, T. S. 2017, J. Plasma Phys., 83, 595830106

Sturrock, P. A. 1958, Phys. Rev., 112, 1488

Sturrock, P. A. 1971, Astrophys. J., 164, 529

Tautz, R. C., Sakai, J.-I., \& Lerche, I. 2007, Astrophysics and Space Science, 310, 159-167

Ursov, V. \& Usov, V. 1988, Astrophysics and Space Science, 140, 325

Usov, V. V. 1987, Astrophys. J., 320, 333

Usov, V. V. 2002, in Neutron Stars, Pulsars, and Supernova Remnants, ed. W. Becker, H. Lesch, \& J. Trümper, 240

Weatherall, J. C. 1994, Astrophys. J., 428, 261 